\title{
ON THE STABILITY OF COMPACT PSEUDO-KÄHLER AND NEUTRAL CALABI-YAU MANIFOLDS
}

\author{
ADELA LATORRE AND LUIS UGARTE
}

\begin{abstract}
We study the stability of compact pseudo-Kähler manifolds, i.e. compact complex manifolds $X$ endowed with a symplectic form compatible with the complex structure of $X$. When the corresponding metric is positive-definite, $X$ is Kähler and any sufficiently small deformation of $X$ admits a Kähler metric by a well-known result of Kodaira and Spencer. We prove that compact pseudo-Kähler surfaces are also stable, but we show that stability fails in every complex dimension $n \geq 3$. Similar results are obtained for compact neutral Kähler and neutral Calabi-Yau manifolds. Finally, motivated by a question of Streets and Tian in the positive-definite case, we construct compact complex manifolds with pseudo-Hermitian-symplectic structures that do not admit any pseudo-Kähler metric.
\end{abstract}

\section{Contents}

1. Introduction

2. Pseudo-Kähler manifolds

2.1. Instability of the pseudo-Kähler property

2.2. Bott-Chern cohomology and stability of pseudo-Kähler manifolds

2.3. Compact pseudo-Kähler surfaces

3. Neutral Calabi-Yau manifolds

3.1. Stability on compact complex surfaces

3.2. A neutral Calabi-Yau nilmanifold in eight dimensions

3.2.1. Counterexamples to a conjecture on pseudo-Kähler nilmanifolds

3.3. Instability in complex dimension $n \geq 4$

4. Pseudo-Hermitian-symplectic structures

Acknowledgments

References

\section{INTRODUCTION}

Let $M$ be an even-dimensional manifold endowed with a complex structure $J$ and a symplectic form $F$. When $J$ and $F$ are compatible, i.e. the symplectic form $F$ is $J$-invariant, and the associated metric $g$ is Riemannian, then the manifold $(M, J, F)$ is Kähler. In the compact Kähler case, the positive-definiteness of $g$ imposes strong topological conditions on the manifold $M$; for instance, its Betti numbers $b_{2 k+1}(M)$ are even and the manifold is formal [15]. Since there are compact complex manifolds with no Kähler metrics, many efforts have been done in understanding the properties of manifolds endowed with a pair $(J, F)$ satisfying weaker conditions than those of a Kähler structure. On the one hand, if we drop the closedness condition for the 2-form $F$, other special Hermitian structures arise, such as the strong Kähler with torsion (SKT) or balanced

Key words and phrases. Complex manifold; symplectic form; pseudo-Kähler metric; neutral Calabi-Yau structure; holomorphic deformation; nilmanifold; solvmanifold. 
Hermitian structures. On the other hand, if we no longer require the positive definiteness of the metric $g$ but preserve the compatibility of the symplectic form $F$ with $J$, then a structure called pseudo-Kähler is obtained.

In this paper we focus on compact manifolds $M$ endowed with a pseudo-Kähler structure, i.e. a pair $(J, F)$ where $J$ is a complex structure and $F$ is a non-degenerate closed 2-form such that $F(J U, J V)=F(U, V)$, for any smooth vector fields $U, V$ on $M$. This is equivalent to $J$ being parallel, i.e. $\nabla J=0$, where $\nabla$ is the Levi-Civita connection of the pseudo-Riemmanian metric $g(U, V)=F(J U, V)$ (see [2]). If the real dimension of $M$ is $2 n$, then the pseudo-Kähler metric has signature $(2 k, 2 n-2 k)$, where $k=n$ corresponds to the Kähler case. In other words, we have a compact complex manifold $X=(M, J)$ with a symplectic form $F$ of bidegree $(1,1)$ with respect to the complex structure $J$. Notice that there are many compact pseudo-Kähler manifolds not admitting any Kähler metric, the simplest example being the compact complex surface known as the Kodaira-Thurston manifold [46.

Pseudo-Kähler structures appear in relation to other interesting structures on manifolds. For instance, compact complex homogeneous manifolds endowed with a pseudo-Kähler structure are classified in [16, 21]. Pseudo-Kähler Einstein metrics on compact complex surfaces are studied by Petean [39]. More recently, it is proved in [25] that there is a natural pseudo-Kähler structure on the universal intermediate $G_{2}$-Jacobian $\mathcal{J}$ of the moduli space of torsion-free $G_{2}$-structures on a fixed compact 7-manifold. Furthermore, any $4 n$-dimensional hypersymplectic manifold in particular has a neutral Calabi-Yau structure [24] (see also [14]), which is a special type of pseudoKähler structure whose underlying metric is Ricci-flat and has signature $(2 n, 2 n)$.

Although several aspects in pseudo-Kähler geometry have been investigated, the stability of these structures under small holomorphic deformations of the complex manifold is, to our knowlegde, only known in the positive-definite case. Indeed, if $X$ is a compact Kähler manifold, then any sufficiently small deformation of $X$ admits a Kähler metric due to a well-known result by Kodaira and Spencer [26]. In this paper we focus on the stability properties of compact pseudo-Kähler manifolds, as well as manifolds with related neutral (Kähler and Calabi-Yau) metrics.

We next explain in more detail the contents of the paper.

In Section 2 we firstly construct compact pseudo-Kähler manifolds of complex dimension $n \geq 3$ that are not stable under small holomorphic deformations of the complex structure. This result motivates the study of sufficient conditions for the stability of the pseudo-Kähler property. Our stability result involves the Bott-Chern cohomology of complex manifolds. We prove that if $X_{0}$ is pseudo-Kähler and the upper-semi-continuous function $t \mapsto h_{\mathrm{BC}}^{1,1}\left(X_{t}\right)$ is constant, then the compact complex manifold $X_{t}$ admits a pseudo-Kähler metric for any small enough $t$ (see Proposition 2.5). Combining this with a result of Teleman [45] on the complex invariant $\Delta^{2}$ introduced by Angella and Tomassini in [6], we prove in Theorem 2.13 that compact pseudo-Kähler surfaces are stable.

The results of Section 2 are illustrated with explicit constructions of pseudo-Kähler nilmanifolds and solvmanifolds, together with their small holomorphic deformations. In particular, we prove in Proposition 2.10 that the Iwasawa manifold and its small deformations do not admit any pseudo-Kähler metric. In contrast, the holomorphically parallelizable Nakamura manifold $X$ is pseudo-Kähler, as proved by Yamada in [50], and there exists a small deformation $X_{\mathbf{t}}$ of $X$ admitting pseudo-Kähler metrics for every $\mathbf{t}$ (see Proposition 2.9).

Section 3 is devoted to a special class of pseudo-Kähler manifolds, namely, neutral Calabi-Yau manifolds. They are neutral Kähler manifolds, i.e. manifolds with even complex dimension $2 m$ and a metric $g$ of signature $(2 m, 2 m)$, that additionally have a nowhere vanishing form $\Phi$ of bidegree $(2 m, 0)$ satisfying $\nabla \Phi=0$, where $\nabla$ is the Levi-Civita connection of $g$. Observe that neutral Calabi-Yau manifolds are Ricci-flat. In Section 3.1 we prove the stability of neutral Kähler and neutral Calabi-Yau structures on compact complex surfaces. However, our Theorem 3.7 shows 
that such structures are not stable on compact complex manifolds of any complex dimension $n \geq 4$. This result is in deep contrast to the case of Kähler Calabi-Yau manifolds, whose deformation space is unobstructed by the well-known Bogomolov-Tian-Todorov theorem [8, 47, 48,

For the proof of Theorem 3.7 we first construct a complex nilmanifold $X$ of complex dimension 4 with (non-flat) neutral Calabi-Yau structures (see Proposition 3.4). An interesting class of neutral Calabi-Yau nilmanifolds, called Kodaira manifolds, was constructed by Fino, Pedersen, Poon and Sørensen in 20. It is worth to note that the complex structure of the nilmanifold $X$ is of a very different kind. Indeed, $X$ has the special feature that it is 4-step and the center of its underlying Lie algebra has minimal dimension, which implies that $X$ is far from being the total space of a torus bundle over a torus. Moreover, in Proposition 3.6 we show that $X$ provides counterexamples to a conjecture in 13 , about the type of invariant complex structures on nilmanifolds that admit compatible pseudo-Kähler metrics. By appropriately deforming the complex nilmanifold $X$, we construct a holomorphic family of compact complex manifolds $\left\{X_{\mathbf{t}}\right\}_{\mathbf{t} \in \Delta}$, with $X_{0}=X$, showing that the neutral Calabi-Yau and neutral Kähler properties are unstable.

In Section 4 we consider pseudo-Hermitian-symplectic structures, which are an indefinite version of Hermitian-symplectic structures. This geometry naturally arises from small deformations of pseudo-Kähler manifolds (see Proposition 4.5). Motivated by a question of Streets and Tian in the positive-definite case [44, Question 1.7], we prove that there are compact complex manifolds admitting pseudo-Hermitian-symplectic structures but no pseudo-Kähler metrics.

\section{PSEUdo-KÄHLER MANIFOLdS}

This section starts showing that compact pseudo-Kähler manifolds of complex dimension $n \geq 3$ are not stable under small holomorphic deformations of the complex structure. This motivates the study of conditions under which a sufficiently small deformation $X_{\mathbf{t}}$ of a pseudo-Kähler manifold $X$ is again pseudo-Kähler. Here, we present a stability result related to the Bott-Chern cohomology of complex manifolds. Several explicit constructions of pseudo-Kähler nilmanifolds and solvmanifolds are provided along the section, illustrating the behaviour of the pseudo-Kähler property under their small holomorphic deformations. In the final part of the section, we focus on the behaviour of small deformations of pseudo-Kähler compact complex surfaces.

Let us recall that a complex analytic family, or holomorphic family, of compact complex manifolds is a proper holomorphic submersion $\pi: \mathcal{X} \longrightarrow \Delta$ between two complex manifolds $\mathcal{X}$ and $\Delta$ [27]. This implies that the fibres $X_{\mathbf{t}}=\pi^{-1}(\mathbf{t})$ are compact complex manifolds of the same dimension. By a classical result of Ehresmann [18, any such family is locally $\mathcal{C}^{\infty}$ trivial (globally, if $\Delta$ is contractible), so all the fibres $X_{\mathbf{t}}$ have the same underlying $\mathcal{C}^{\infty}$ manifold $M$. Consequently, the holomorphic family can be viewed as a collection $\left\{X_{\mathbf{t}}\right\}_{\mathbf{t} \in \Delta}$ of complex manifolds $X_{\mathbf{t}}=\left(M, J_{\mathbf{t}}\right)$, where $J_{\mathbf{t}}$ is the complex structure of $X_{\mathbf{t}}$ for $\mathbf{t} \in \Delta$.

A classical result of Kodaira and Spencer [26] asserts that the property of being a compact Kähler manifold is stable under holomorphic deformations. In the following section we prove that such a stability result cannot be extended to compact pseudo-Kähler manifolds.

2.1. Instability of the pseudo-Kähler property. Here we prove that compact pseudo-Kähler manifolds of complex dimension $n \geq 3$ are in general not stable under small deformations of the complex structure. In the proof we will consider an appropriate holomorphic family consisting of complex nilmanifolds. We recall that a nilmanifold is a compact quotient $N=\Gamma \backslash G$ of a connected and simply connected nilpotent Lie group $G$ by a lattice $\Gamma$ of maximal rank in $G$. A complex nilmanifold $X=(N, J)$ is a nilmanifold $N=\Gamma \backslash G$ endowed with an invariant complex structure $J$, i.e. $J$ comes from a left-invariant complex structure on $G$ by passing to the quotient $\Gamma \backslash G$. (For results on complex nilmanifolds see for instance [3, 11, 41] and the references therein.) 
Proposition 2.1. There is a holomorphic family of compact complex manifolds $\left\{X_{\mathbf{t}}\right\}_{\mathbf{t} \in \Delta}$ of complex dimension 3 , where $\Delta=\{\mathbf{t} \in \mathbb{C}|| \mathbf{t} \mid<1\}$, such that:

(i) $X_{0}$ is a pseudo-Kähler manifold;

(ii) $X_{\mathbf{t}}$ does not admit pseudo-Kähler metrics for any $\mathbf{t} \neq 0$.

Hence, the pseudo-Kähler property is not stable under deformations of the complex structure.

Proof. Let $X$ be the complex nilmanifold defined by the following complex structure equations

$$
d \omega^{1}=d \omega^{2}=0, \quad d \omega^{3}=\omega^{1 \overline{2}} .
$$

Here $\omega^{k}$ has bidegree $(1,0)$ and $\omega^{j \bar{k}}$ denotes the $(1,1)$-form $\omega^{j} \wedge \overline{\omega^{k}}$.

Observe that the compact complex manifold $X$ has pseudo-Kähler metrics. For instance, the 2 -form

$$
F=i \omega^{1 \overline{1}}+\omega^{2 \overline{3}}-\omega^{3 \overline{2}}
$$

satisfies that $F=\bar{F}$, i.e. the 2 -form $F$ is real, and $F^{3} \neq 0$, i.e. it is non-degenerate. The form $F$ is compatible with the complex structure $J$ of $X$ because it is of pure type $(1,1)$ on $X$. Moreover, from (11) we get that $d F=0$, so the form $F$ is symplectic. Thus, $F$ defines a pseudo-Kähler metric on $X$.

Notice that the $(0,1)$-form $\omega^{\overline{3}}$ is $\bar{\partial}$-closed on $X$. We will use the Dolbeault cohomology class $\left[\omega^{\overline{3}}\right] \in H_{\bar{\partial}}^{0,1}(X)$ to perform an appropriate holomorphic deformation of $X$. For each $\mathbf{t} \in \mathbb{C}$ such that $|\mathbf{t}|<1$, let us consider the complex nilmanifold $X_{\mathbf{t}}$ defined by the following complex basis of (1,0)-forms:

$$
\omega_{\mathbf{t}}^{1}:=\omega^{1}, \quad \omega_{\mathbf{t}}^{2}:=\omega^{2}, \quad \omega_{\mathbf{t}}^{3}:=\omega^{3}+\mathbf{t} \omega^{\overline{3}} .
$$

It follows from (11) and (2) that the complex structure equations for $X_{\mathbf{t}}$ are

$$
d \omega_{\mathbf{t}}^{1}=d \omega_{\mathbf{t}}^{2}=0, \quad d \omega_{\mathbf{t}}^{3}=\omega_{\mathbf{t}}^{1 \overline{2}}-\mathbf{t} \omega_{\mathbf{t}}^{2 \overline{1}} .
$$

In order to prove that $X_{\mathbf{t}}$ has no pseudo-Kähler metrics for any $\mathbf{t} \neq 0$, we use Nomizu's theorem [36] for the de Rham cohomology of nilmanifolds together with the symmetrization process introduced in [7]. More concretely, we take into account that, by [49, Remark 5], any closed $k$-form $\alpha$ on a nilmanifold is cohomologous to the invariant $k$-form $\widetilde{\alpha}$ obtained by the symmetrization process.

Fix any $\mathbf{t} \in \Delta$. Since the complex structure on $X_{\mathbf{t}}$ is invariant, if there exists a pseudo-Kähler structure $\Theta$ on $X_{\mathbf{t}}$, then the form $\widetilde{\Theta}$, obtained by symmetrization of $\Theta$, would be an invariant closed real 2-form of bidegree $(1,1)$ such that $[\widetilde{\Theta}]=[\Theta]$ in the second de Rham cohomology group $H_{\mathrm{dR}}^{2}\left(X_{\mathbf{t}} ; \mathbb{R}\right)$. In particular, this would imply $\left[\widetilde{\Theta}^{3}\right]=[\Theta]^{3} \neq 0$ in the de Rham cohomology group $H_{\mathrm{dR}}^{6}\left(X_{\mathbf{t}} ; \mathbb{R}\right)$, due to the non-degeneracy of $\Theta$ on the compact complex manifold $X_{\mathbf{t}}$. However, for $\mathbf{t} \neq 0$, it follows from (3) that any invariant closed (1,1)-form $\zeta$ on $X_{\mathbf{t}}$ satisfies

$$
\zeta \in \mathbb{C}\left\langle\omega_{\mathbf{t}}^{1 \overline{1}}, \omega_{\mathbf{t}}^{1 \overline{2}}, \omega_{\mathbf{t}}^{2 \overline{1}}, \omega_{\mathbf{t}}^{2 \overline{2}}\right\rangle
$$

which implies that $\zeta^{3}=\zeta \wedge \zeta \wedge \zeta=0$, i.e. $\zeta$ is degenerate. In conclusion, there are no (invariant or not) pseudo-Kähler metrics on $X_{\mathbf{t}}$ for $\mathbf{t} \neq 0$.

Remark 2.2. Let us consider $Y_{\mathbf{t}}=X_{\mathbf{t}} \times \mathbb{T}^{m}$, where $\left\{X_{\mathbf{t}}\right\}_{\mathbf{t} \in \Delta}$ is the holomorphic family given in Proposition 2.1 and $\mathbb{T}^{m}$ denotes the $m$-dimensional complex torus endowed with its standard Kähler metric. A similar argument as the one in the proof of the previous proposition shows that $Y_{\mathbf{t}}$ does not admit any pseudo-Kähler metric for $\mathbf{t} \neq 0$. Hence, the pseudo-Kähler property is not stable in any complex dimension $n \geq 3$ (see Theorem 3.7 for an irreducible example in complex dimension 4). In contrast, we will prove in Section 2.3 that compact pseudo-Kähler surfaces are stable. 
The following example illustrates that, although the pseudo-Kähler property is in general not stable, one can find certain deformations where the existence of pseudo-Kähler metrics is preserved.

Example 2.3. Let us consider the differentiable family $\left\{X_{t}\right\}_{t \in(-1,1)}$ of compact complex nilmanifolds defined by the complex structure equations

$$
d \omega_{t}^{1}=0, \quad d \omega_{t}^{2}=\omega_{t}^{1 \overline{1}}, \quad d \omega_{t}^{3}=\omega_{t}^{12}+t \omega_{t}^{1 \overline{2}} .
$$

We note that this is a differentiable family of deformations of the compact complex nilmanifold $X=X_{0}$ determined by the equations

$$
d \omega^{1}=0, \quad d \omega^{2}=\omega^{1 \overline{1}}, \quad d \omega^{3}=\omega^{12} .
$$

The manifolds $X_{t}$ in this family are all pseudo-Kähler since, for instance, $F_{t}=i\left(\omega_{t}^{1 \overline{3}}+\omega_{t}^{3 \overline{1}}\right)+$ $i(1+t) \omega_{t}^{2 \overline{2}}$ defines a pseudo-Kähler metric on $X_{t}$ for every $t \in(-1,1)$.

Hence, one would like to study additional conditions under which the pseudo-Kähler property becomes stable. We next establish a condition in terms of the Bott-Chern cohomology.

2.2. Bott-Chern cohomology and stability of pseudo-Kähler manifolds. Here, we show that the stability of the pseudo-Kähler property is closely related to the variation of the BottChern cohomology. We recall that the Bott-Chern and the Aeppli cohomologies [1, 9] (see also [3]) of a compact complex manifold $X$ are defined, respectively, by

$$
H_{\mathrm{BC}}^{\bullet, \bullet}(X):=\frac{\operatorname{ker} \partial \cap \operatorname{ker} \bar{\partial}}{\operatorname{im} \partial \bar{\partial}} \quad \text { and } \quad H_{\mathrm{A}}^{\bullet, \bullet}(X):=\frac{\operatorname{ker} \partial \bar{\partial}}{\operatorname{im} \partial+\operatorname{im} \bar{\partial}} .
$$

The dimensions of these cohomology groups will be denoted by $h_{\mathrm{BC}}^{p, q}(X)=\operatorname{dim}_{\mathbb{C}} H_{\mathrm{BC}}^{p, q}(X)$ and $h_{\mathrm{A}}^{p, q}(X)=\operatorname{dim}_{\mathbb{C}} H_{\mathrm{A}}^{p, q}(X)$.

Suppose that $X$ admits a pseudo-Kähler metric defined by $F$. Since the real form $F^{k}$ is closed and has bidegree $(k, k)$, it defines a Bott-Chern class $\left[F^{k}\right] \in H_{\mathrm{BC}}^{k, k}(X)$ for any $1 \leq k \leq n$. Moreover:

Lemma 2.4. Let $X$ be a compact complex manifold with $\operatorname{dim}_{\mathbb{C}} X=n$. If $X$ admits a pseudoKähler metric, then $h_{\mathrm{BC}}^{k, k}(X) \geq 1$ for any $1 \leq k \leq n$.

Proof. Let us consider a pseudo-Kähler metric on $X$ defined by $F$ and suppose that the class $\left[F^{k}\right] \in H_{\mathrm{BC}}^{k, k}(X)$ is zero for some $1 \leq k \leq n$. This fact implies that $F^{k}=\partial \bar{\partial} \beta$ for some form $\beta \in \Omega^{k-1, k-1}(X)$, so

$$
F^{n}=F^{n-k} \wedge F^{k}=F^{n-k} \wedge \partial \bar{\partial} \beta=\partial\left(F^{n-k} \wedge \bar{\partial} \beta\right)=d\left(F^{n-k} \wedge \bar{\partial} \beta\right),
$$

which contradits the non-degeneracy of the form $F$.

The following proposition is an extension of the Kodaira-Spencer stability result [26, Theorem 15] for Kähler metrics. We recall that, for every $(p, q)$, the function $t \mapsto h_{\mathrm{BC}}^{p, q}\left(X_{t}\right)$ is uppersemi-continuous [42].

Proposition 2.5. Let $X$ be a compact pseudo-Kähler manifold, and let $\left\{X_{t}\right\}_{t \in(-\varepsilon, \varepsilon)}$ be a differentiable family of deformations of $X=X_{0}$, where $\varepsilon>0$. Suppose that the upper-semi-continuous function $t \mapsto h_{\mathrm{BC}}^{1,1}\left(X_{t}\right)$ is constant. Then, the compact complex manifold $X_{t}$ admits a pseudoKähler metric for any $t$ close enough to 0 .

Proof. Let $\left\{\omega_{t}\right\}_{t}$ be a family of Hermitian metrics on $X_{t}$ for $t \in(-\varepsilon, \varepsilon)$. For each $t$, we consider the Bott-Chern Laplacian $\Delta_{t}^{\mathrm{BC}}$ associated to the Hermitian metric $\omega_{t}$ on $X_{t}$ and the corresponding Green operator $G_{t}$ [42. Denote by $H_{t}: \Omega_{\mathbb{C}}^{*}\left(X_{t}\right) \rightarrow$ ker $\Delta_{t}^{\mathrm{BC}}$ the projection onto the space of harmonic forms with respect to $\Delta_{t}^{\mathrm{BC}}$ (and with respect to the $L_{\omega_{t}}^{2}$-orthogonal decomposition 
induced by $\omega_{t}[42]$ ), and by $\pi_{t}^{1,1}: \Omega_{\mathbb{C}}^{*}(X) \rightarrow \Omega^{1,1}\left(X_{t}\right)$ the projection of the space of complex forms on $X$ onto the space of $(1,1)$-forms on $X_{t}$.

Now, for any $t \in(-\varepsilon, \varepsilon)$, the operator $\Pi_{t}$ defined by

$$
\Pi_{t}:=\left(H_{t}+\partial_{t} \bar{\partial}_{t}\left(\partial_{t} \bar{\partial}_{t}\right)^{* t} G_{t}\right) \circ \pi_{t}^{1,1}: \Omega_{\mathbb{C}}^{*}(X) \longrightarrow \operatorname{ker} \partial_{t} \cap \operatorname{ker} \bar{\partial}_{t}
$$

gives the projection of the space of complex forms on $X$ onto the space of $\partial_{t}$-closed and $\bar{\partial}_{t}$-closed $(1,1)$-forms on the compact complex manifold $X_{t}$. Here, $*_{t}$ is the Hodge-operator with respect to the Hermitian metric $\omega_{t}$ on $X_{t}$. Since the function $t \mapsto h_{\mathrm{BC}}^{1,1}\left(X_{t}\right)$ is constant, by elliptic theory [26. Theorem 7] one has that the family $\left\{\Pi_{t}\right\}_{t}$ is smooth in $t$. Let $F_{0}$ be a pseudo-Kähler metric on $X_{0}=X$. For $t \in(-\varepsilon, \varepsilon)$, we set

$$
F_{t}:=\frac{\Pi_{t} F_{0}+\overline{\Pi_{t} F_{0}}}{2}
$$

Then, the family $\left\{F_{t}\right\}_{t}$ is smooth in $t$, and each $F_{t}$ is a real (1,1)-form on $X_{t}$ which is $d$-closed, because it is closed by $\partial_{t}$ and $\bar{\partial}_{t}$. Since $F_{0}^{n}$ is a nowhere vanishing $(n, n)$-form, we have that $F_{t}$ is non-degenerate for $t$ close enough to 0 . Therefore, the form $F_{t}$ defines a pseudo-Kähler metric on the compact complex manifold $X_{t}$ for any $t$ close enough to 0 .

Example 2.6. We here show that for the holomorphic family $\left\{X_{\mathbf{t}}\right\}_{\mathbf{t} \in \Delta}$ constructed in the proof of Proposition 2.1, the upper-semi-continuous function $\mathbf{t} \mapsto h_{\mathrm{BC}}^{1,1}\left(X_{\mathbf{t}}\right)$ is not constant. According to Proposition 2.5. this fact gives a reason for the instability of the pseudo-Kähler property along the deformation of $X_{0}$. Indeed, for the compact complex manifold $X_{0}$ we have

$$
H_{\mathrm{BC}}^{1,1}\left(X_{0}\right)=\left\langle\left[i \omega^{1 \overline{1}}\right],\left[i \omega^{2 \overline{2}}\right],\left[\omega^{1 \overline{2}}-\omega^{2 \overline{1}}\right],\left[i\left(\omega^{1 \overline{2}}+\omega^{2 \overline{1}}\right)\right],\left[\omega^{2 \overline{3}}-\omega^{3 \overline{2}}\right],\left[i\left(\omega^{2 \overline{3}}+\omega^{3 \overline{2}}\right)\right]\right\rangle,
$$

and, for any $\mathbf{t} \in \Delta-\{0\}$, the Bott-Chern cohomology group of bidegree $(1,1)$ of $X_{\mathbf{t}}$ is

$$
H_{\mathrm{BC}}^{1,1}\left(X_{\mathbf{t}}\right)=\left\langle\left[i \omega_{\mathbf{t}}^{1 \overline{1}}\right],\left[i \omega_{\mathbf{t}}^{2 \overline{2}}\right],\left[\omega_{\mathbf{t}}^{1 \overline{2}}-\omega_{\mathbf{t}}^{2 \overline{1}}\right],\left[i\left(\omega_{\mathbf{t}}^{1 \overline{2}}+\omega_{\mathbf{t}}^{2 \overline{1}}\right)\right]\right\rangle .
$$

Therefore, $h_{\mathrm{BC}}^{1,1}\left(X_{0}\right)=6$, and $h_{\mathrm{BC}}^{1,1}\left(X_{\mathbf{t}}\right)=4$, for any $\mathbf{t} \neq 0$.

Example 2.7. Let us consider the differentiable family $\left\{X_{t}\right\}_{t \in(-1,1)}$ of compact pseudo-Kähler manifolds given in Example 2.3. The Bott-Chern cohomology group of bidegree $(1,1)$ of $X_{t}$ is

$$
H_{\mathrm{BC}}^{1,1}\left(X_{t}\right)=\left\langle\left[i \omega_{t}^{1 \overline{1}}\right],\left[\omega_{t}^{1 \overline{2}}-\omega_{t}^{2 \overline{1}}\right],\left[i\left(\omega_{t}^{1 \overline{2}}+\omega_{t}^{2 \overline{1}}\right)\right],\left[i\left(\omega_{t}^{1 \overline{3}}+\omega_{t}^{3 \overline{1}}\right)+i(1+t) \omega_{t}^{2 \overline{2}}\right]\right\rangle .
$$

Therefore, $h_{\mathrm{BC}}^{1,1}\left(X_{t}\right)=4$ for every $t$, i.e. the function $t \mapsto h_{\mathrm{BC}}^{1,1}\left(X_{t}\right)$ is constant.

We next show that the result in Example 2.7 can indeed be extended to any family of deformations of $X=X_{0}$.

Proposition 2.8. Let $X$ be the compact pseudo-Kähler manifold in Example 2.3 defined by (44), and let $\left\{X_{t}\right\}_{t \in(-\varepsilon, \varepsilon)}$ be any differentiable family of deformations of $X=X_{0}$, where $\epsilon>0$. Then, $X_{t}$ admits a pseudo-Kähler metric for every $t$ close enough to 0.

Proof. Since $X$ is a nilmanifold endowed with an invariant complex structure, one has by 40 , Theorem 2.6] that the complex structure $J_{t}$ of any sufficiently small deformation $X_{t}$ of $X$ is also invariant. The dimension of the Bott-Chern cohomology groups of any invariant complex structure $J_{t}$ is given in [4, Table 2] and [30, Appendix 6]. Note that the Lie algebra underlying the nilmanifold $X$ is precisely $\mathfrak{h}_{15}$, and $h_{\mathrm{BC}}^{1,1}\left(\mathfrak{h}_{15}, J\right) \geq 4$ for any complex structure $J$ on $\mathfrak{h}_{15}$.

Since $h_{\mathrm{BC}}^{1,1}\left(X_{t}\right)=h_{\mathrm{BC}}^{1,1}\left(\mathfrak{h}_{15}, J_{t}\right) \geq 4$ varies upper-semi-continuously along differentiable families [42], for any $t$ close enough to 0 we have

$$
4=h_{\mathrm{BC}}^{1,1}\left(X_{0}\right) \geq h_{\mathrm{BC}}^{1,1}\left(X_{t}\right) \geq 4,
$$


and thus $h_{\mathrm{BC}}^{1,1}\left(X_{t}\right)=4$. Hence, the function $t \mapsto h_{\mathrm{BC}}^{1,1}\left(X_{t}\right)$ is constant and, by Proposition 2.5, $X_{t}$ admits a pseudo-Kähler metric for any $t$ close enough to 0 .

We observe that the condition on $h_{\mathrm{BC}}^{1,1}$ given in Proposition 2.5 is sufficient but not necessary. To illustrate this fact, we next study the existence of pseudo-Kähler metrics on the small deformations of the well-known Nakamura manifold. This manifold is a holomorphically parallelizable solvmanifold, i.e. a compact quotient $X=\Gamma \backslash G$ where $G$ is a simply connected complex solvable Lie group and $\Gamma$ a lattice in $G$.

Let $G$ be the simply-connected complex solvable Lie group given by

$$
G=\left\{\left(\begin{array}{cccc}
e^{z_{1}} & 0 & 0 & z_{2} \\
0 & e^{-z_{1}} & 0 & z_{3} \\
0 & 0 & 1 & z_{1} \\
0 & 0 & 0 & 1
\end{array}\right) \quad \mid z_{1}, z_{2}, z_{3} \in \mathbb{C}\right\}
$$

i.e. $G$ is the semi-direct product $G=\mathbb{C} \ltimes_{\varphi} \mathbb{C}^{2}$, where $\left(z_{2}, z_{3}\right)$ are the coordinates on $\mathbb{C}^{2}$ and

$$
\varphi\left(z_{1}\right)=\left(\begin{array}{cc}
e^{z_{1}} & 0 \\
0 & e^{-z_{1}}
\end{array}\right), \quad z_{1} \in \mathbb{C} .
$$

One can consider the symplectic form on the Lie group $G$ defined by

$$
F=i d z_{1} \wedge d \bar{z}_{1}+d z_{2} \wedge d \bar{z}_{3}+d \bar{z}_{2} \wedge d z_{3}
$$

which clearly has bidegree $(1,1)$ with respect to the complex structure of $G$. That is to say, $F$ is a pseudo-Kähler metric on $G$. Notice that $F$ is not left-invariant on $G$, indeed the forms

$$
\omega^{1}=d z_{1}, \quad \omega^{2}=e^{-z_{1}} d z_{2}, \quad \omega^{3}=e^{z_{1}} d z_{3},
$$

constitute a basis of left-invariant forms of bidegree $(1,0)$ on the complex Lie group $G$.

Yamada proved in [50] that there is a lattice $\Gamma$ of maximal rank in $G$ such that $F$ descends to a pseudo-Kähler metric on $X=\Gamma \backslash G$. Let $A \in \mathrm{SL}(2, \mathbb{Z})$ be a unimodular matrix with distinct real eigenvalues $\lambda$ and $\lambda^{-1}$, and take $a=\log \lambda$. One can consider a lattice $\Gamma$ in $G$ of the form $\Gamma=\Gamma_{1} \ltimes_{\varphi} \Gamma_{\mathbb{C}^{2}}$, with $\Gamma_{1}=a \mathbb{Z}+2 \pi i \mathbb{Z}$ and $\Gamma_{\mathbb{C}^{2}}$ a lattice in $\mathbb{C}^{2}$. The compact complex (solv)manifold $X=\Gamma \backslash G$ is known as the (holomorphically parallelizable) Nakamura manifold [35]. Yamada proved in [50, Theorem 2.1] that the symplectic form $F$ given in (5) descends to $X$, providing in this way the first example of a non-toral compact holomorphically parallelizable pseudo-Kähler solvmanifold. In fact, in terms of the (1,0)-basis $\left\{\omega^{k}\right\}_{k=1}^{3}$, the form $F$ expresses as

$$
F=i \omega^{1} \wedge \omega^{\overline{1}}+e^{2 i \mathfrak{I m} z_{1}} \omega^{2} \wedge \omega^{\overline{3}}+e^{-2 i \mathfrak{I m} z_{1}} \omega^{\overline{2}} \wedge \omega^{3},
$$

where the functions $e^{2 i \mathfrak{I m} z_{1}}$ and $e^{-2 i \mathfrak{I m} z_{1}}$ are $\Gamma$-invariant. Therefore, $F$ induces a pseudo-Kähler metric on the Nakamura manifold $X$.

In 22] Hasegawa extended Yamada's result to any compact holomorphically parallelizable solvmanifold of complex dimension 3, determining all the lattices of simply-connected unimodular complex solvable Lie groups. Hasegawa proves that such a complex solvmanifold admits a pseudoKähler metric if and only if the Hodge number $h_{\bar{\partial}}^{0,1}=3[22$, Theorem 2]. Notice that for the Nakamura manifold $X$ one has $H_{\bar{\partial}}^{0,1}(X)=\left\langle\left[\omega^{\overline{1}}\right],\left[e^{-2 i \mathfrak{I m} z_{1}} \omega^{\overline{2}}\right],\left[e^{2 i \mathfrak{I m} z_{1}} \omega^{\overline{3}}\right]\right\rangle$.

In the following proposition we show that there is a small deformation $X_{\mathbf{t}}$ of $X$ admitting pseudo-Kähler metrics. Note that by [5] the Nakamura manifold has $h_{\mathrm{BC}}^{1,1}(X)=7$, whereas our small deformation satisfies $h_{\mathrm{BC}}^{1,1}\left(X_{\mathbf{t}}\right)=3$ for every $\mathbf{t} \neq 0$.

Proposition 2.9. There exists a small deformation $X_{\mathbf{t}}$ of the holomorphically parallelizable Nakamura manifold $X$ admitting pseudo-Kähler metrics for any $\mathbf{t}$. 
Proof. In [5, Section 4] Angella and Kasuya studied some deformations of the Nakamura manifold $X=\Gamma \backslash G$. We here consider the deformation given by $\mathbf{t} \frac{\partial}{\partial z_{1}} \otimes d \bar{z}_{1} \in H^{0,1}\left(X ; T^{1,0} X\right)$, which corresponds to the case (1) in their paper.

Note that this deformation defines a holomorphic family $\left\{X_{\mathbf{t}}\right\}_{\mathbf{t} \in \Delta}$, where $\Delta=\{\mathbf{t} \in \mathbb{C}|| \mathbf{t} \mid<1\}$, such that $X_{0}=X$. We have the $(1,0)$-forms on $X_{\mathbf{t}}$ given by

$$
\omega_{\mathbf{t}}^{1}=d z_{1}-\mathbf{t} d \bar{z}_{1}, \quad \omega_{\mathbf{t}}^{2}=e^{-z_{1}} d z_{2}, \quad \omega_{\mathbf{t}}^{3}=e^{z_{1}} d z_{3},
$$

whose differentials satisfy

$$
\left\{\begin{array}{l}
d \omega_{\mathbf{t}}^{1}=0, \\
d \omega_{\mathbf{t}}^{2}=-\frac{1}{1-|\mathbf{t}|^{2}} \omega_{\mathbf{t}}^{12}+\frac{\mathbf{t}}{1-|\mathbf{t}|^{2}} \omega_{\mathbf{t}}^{2 \overline{1}}, \\
d \omega_{\mathbf{t}}^{3}=\frac{1}{1-|\mathbf{t}|^{2}} \omega_{\mathbf{t}}^{13}-\frac{\mathbf{t}}{1-|\mathbf{t}|^{2}} \omega_{\mathbf{t}}^{3 \overline{1}} .
\end{array}\right.
$$

A direct calculation shows that the real 2-form $F_{\mathbf{t}}$ defined on $X_{\mathbf{t}}$ by

$$
F_{\mathbf{t}}=i \omega_{\mathbf{t}}^{1 \overline{1}}+e^{2 i \mathfrak{I m} z_{1}} \omega_{\mathbf{t}}^{2 \overline{3}}+e^{-2 i \mathfrak{I m} z_{1}} \omega_{\mathbf{t}}^{\overline{2} 3}
$$

is closed and non-degenerate. Since $F_{\mathbf{t}}$ has bidegree $(1,1)$ with respect to the complex structure on $X_{\mathbf{t}}$, we get a pseudo-Kähler metric on the compact complex manifold $X_{\mathbf{t}}$, for any $\mathbf{t} \in \Delta$.

In complex dimension 3, there is another well-known complex holomorphically parallelizable solvmanifold, namely, the Iwasawa (nil)manifold. Although this manifold is symplectic, it is proved in [12, Theorem 3.2] (see also [50]) that a holomorphically parallelizable nilmanifold is pseudo-Kähler if and only if it is a complex torus. Hence, the Iwasawa manifold does not admit pseudo-Kähler metrics. Moreover:

Proposition 2.10. The Iwasawa manifold and its small deformations do not admit any pseudoKähler metric.

Proof. Recall that the Iwasawa manifold $X$ is the compact complex manifold obtained as a quotient of the 3-dimensional complex Heisenberg group. As we have previously stated, by [12, Theorem 3.2] the Iwasawa manifold is not pseudo-Kähler. Nakamura studied in [35] the small deformations of the Iwasawa manifold (see also [3] for more details). It turns out that the complex structure equations of any sufficiently small deformation $X_{\mathbf{t}}$ of the Iwasawa manifold $X=X_{0}$ can be written as

$$
\left\{\begin{array}{l}
d \omega_{\mathbf{t}}^{1}=d \omega_{\mathbf{t}}^{2}=0 \\
d \omega_{\mathbf{t}}^{3}=\sigma_{12} \omega_{\mathbf{t}}^{12}+\sigma_{1 \overline{1}} \omega_{\mathbf{t}}^{1 \overline{1}}+\sigma_{1 \overline{2}} \omega_{\mathbf{t}}^{1 \overline{2}}+\sigma_{2 \overline{1}} \omega_{\mathbf{t}}^{2 \overline{1}}+\sigma_{2 \overline{2}} \omega_{\mathbf{t}}^{2 \overline{2}},
\end{array}\right.
$$

where the coefficients $\sigma_{12}, \sigma_{1 \overline{1}}, \sigma_{1 \overline{2}}, \sigma_{2 \overline{1}}, \sigma_{2 \overline{2}} \in \mathbb{C}$ only depend on the parameter $\mathbf{t}$ in the deformation space $\Delta=\left\{\mathbf{t}=\left(t_{11}, t_{12}, t_{21}, t_{22}, t_{31}, t_{32}\right) \in \mathbb{C}^{6}|| \mathbf{t} \mid<\varepsilon\right\}$ for a sufficiently small $\varepsilon>0$. Let $H^{+}\left(X_{\mathbf{t}}\right) \subset H_{\mathrm{dR}}^{2}\left(X_{\mathbf{t}} ; \mathbb{R}\right)$ be the subspace determined by the second de Rham cohomology classes that can be represented by closed real forms of bidegree $(1,1)$ on the compact complex manifold $X_{\mathbf{t}}$. As proved in [29, Proposition 3.4], this subspace is given by

$$
H^{+}\left(X_{\mathbf{t}}\right)=\left\langle\left[i \omega_{\mathbf{t}}^{1 \overline{1}}\right],\left[i \omega_{\mathbf{t}}^{2 \overline{2}}\right],\left[\omega_{\mathbf{t}}^{1 \overline{2}}-\omega_{\mathbf{t}}^{2 \overline{1}}\right],\left[i\left(\omega_{\mathbf{t}}^{1 \overline{2}}+\omega_{\mathbf{t}}^{2 \overline{1}}\right)\right]\right\rangle .
$$

It is then clear that any de Rham cohomology class in $H^{+}\left(X_{\mathbf{t}}\right)$ is degenerate, so $X_{\mathbf{t}}$ does not admit any pseudo-Kähler metric. 
2.3. Compact pseudo-Kähler surfaces. We start this section applying our Proposition 2.5 to provide a stability result for the pseudo-Kähler property in terms of the complex invariant $\Delta^{2}(X)$ introduced in 6].

Let us recall that, for each $k \in \mathbb{N}$, Angella and Tomassini introduced the complex invariant

$$
\Delta^{k}(X):=\sum_{p+q=k}\left(h_{\mathrm{BC}}^{p, q}(X)+h_{A}^{p, q}(X)\right)-2 b_{k},
$$

which is a non-negative integer [6, Theorem A]. Furthermore, by [6, Theorem B], a compact complex manifold $X$ satisfies the $\partial \bar{\partial}$-Lemma if and only if $\Delta^{k}(X)=0$ for any $k$. We are interested in the term $\Delta^{2}(X)$.

Corollary 2.11. Let $X$ be a compact pseudo-Kähler manifold, and let $\left\{X_{t}\right\}_{t \in(-\varepsilon, \varepsilon)}$ be a differentiable family of deformations of $X=X_{0}$, where $\varepsilon>0$. If the upper-semi-continuous function $t \mapsto \Delta^{2}\left(X_{t}\right)$ is constant, then $X_{t}$ admits a pseudo-Kähler metric for any $t$ close enough to 0 .

Proof. Suppose $\Delta^{2}\left(X_{t}\right)=c$ for any $t \in(-\varepsilon, \varepsilon)$, where $c$ is a non-negative integer. Then, expanding (66) for $k=2$, we have

$$
\begin{aligned}
& h_{\mathrm{BC}}^{2,0}\left(X_{0}\right)+h_{\mathrm{BC}}^{1,1}\left(X_{0}\right)+h_{\mathrm{BC}}^{0,2}\left(X_{0}\right)+h_{A}^{2,0}\left(X_{0}\right)+h_{A}^{1,1}\left(X_{0}\right)+h_{A}^{0,2}\left(X_{0}\right) \\
& =\Delta^{2}\left(X_{0}\right)+2 b_{2}=c+2 b_{2}=\Delta^{2}\left(X_{t}\right)+2 b_{2} \\
& =h_{\mathrm{BC}}^{2,0}\left(X_{t}\right)+h_{\mathrm{BC}}^{1,1}\left(X_{t}\right)+h_{\mathrm{BC}}^{0,2}\left(X_{t}\right)+h_{A}^{2,0}\left(X_{t}\right)+h_{A}^{1,1}\left(X_{t}\right)+h_{A}^{0,2}\left(X_{t}\right) .
\end{aligned}
$$

Since the functions $t \mapsto h_{\mathrm{BC}}^{p, q}\left(X_{t}\right)$ and $t \mapsto h_{A}^{p, q}\left(X_{t}\right)$ are upper-semi-continuous for any $(p, q)$, they all must be constant. In particular, the function $t \mapsto \operatorname{dim}_{\mathbb{C}} H_{\mathrm{BC}}^{1,1}\left(X_{t}\right)$ is constant, so Proposition 2.5 implies that the compact complex manifold $X_{t}$ admits a pseudo-Kähler metric for any $t$ close enough to 0 .

The following result comes as a direct consequence.

Corollary 2.12. Any sufficiently small deformation of a compact pseudo-Kähler manifold $X$ satisfying $\Delta^{2}(X)=0$ admits a pseudo-Kähler metric. In particular, any sufficiently small deformation of a compact pseudo-Kähler $\partial \bar{\partial}$-manifold is pseudo-Kähler.

Let us recall that a compact complex surface is Kähler if and only if its first Betti number $b_{1}$ is even (see Kodaira's classification of surfaces, 34] and [43, or [10, 28, for a direct proof). To our knowledge, there is no classification of compact complex surfaces admitting a pseudo-Kähler metric. Petean found in [39] some obstructions to the existence of indefinite Kähler metrics on compact complex surfaces in terms of Seiberg-Witten invariants. Here "indefinite Kähler" means that the signature of the metric is $(2,2)$, i.e. the metric is pseudo-Kähler but non-Kähler (see [39, Theorem 4] for a list of the possible compact complex surfaces that might admit an indefinite Kähler metric).

Next, we make use of our previous results and a result by Teleman [45] to prove the stability of the pseudo-Kähler property on compact complex surfaces.

Theorem 2.13. Any sufficiently small deformation of a compact pseudo-Kähler surface admits a pseudo-Kähler metric.

Proof. Let $X$ be a compact pseudo-Kähler surface. By Teleman's result [45, Lemma 2.3], the invariant $\Delta^{2}(X)$ is either 0 or 2. Moreover, Teleman also proves that $\Delta^{2}(X)=0$ if and only if the first Betti number $b_{1}$ is even, which is equivalent to the existence of a Kähler metric on $X$. Hence, in the case $\Delta^{2}(X)=0$, any sufficiently small deformation of $X$ is again Kähler by [26], so in particular pseudo-Kähler. 
Let us now focus on the case $\Delta^{2}(X)=2$. By 45 this is equivalent to $X$ having odd first Betti number, so the condition $\Delta^{2}(X)=2$ is a topological property. Thus, $\Delta^{2}\left(X_{t}\right)=2$ for any differentiable family $\left\{X_{t}\right\}_{t \in(-\varepsilon, \varepsilon)}$ of deformations of the compact pseudo-Kähler surface $X=X_{0}$. Since the upper-semi-continuous function $t \mapsto \Delta^{2}\left(X_{t}\right)$ is constant, by Corollary 2.11 the compact complex surface $X_{t}$ admits a pseudo-Kähler metric for any $t$ close enough to 0 .

To finish this section we show that there is only one compact complex non-Kähler surface diffeomorphic to a solvmanifold that admits pseudo-Kähler metrics, namely the Kodaira-Thurston manifold.

Hasegawa classified in 23 the compact complex surfaces $X$ that are diffeomorphic to a 4dimensional solvmanifold. Moreover, he proved that the complex structures on such surfaces are invariant (see [37 for a study of 4-dimensional Lie algebras admitting pseudo-Kähler metrics). In fact, in [23, Theorem 1] it is shown that $X$ must be one of the following surfaces: complex torus, hyperelliptic surface, Inoue surface of type $\mathcal{S}_{M}$, primary Kodaira surface, secondary Kodaira surface, or Inoue surface of type $\mathcal{S}^{ \pm}$. Only the first two are Kähler, whereas the other ones have vanishing second Betti number, with the only exception of a primary Kodaira surface. It is wellknown that the latter admits symplectic forms [46]. Consequently, a compact complex non-Kähler surface diffeomorphic to a solvmanifold admits a symplectic form if and only if it is a primary Kodaira surface.

We recall that a primary Kodaira surface, which we will denote by $K T$, admits pseudo-Kähler metrics. By [23], for any complex structure on $K T$ there is a global basis $\left\{\omega^{1}, \omega^{2}\right\}$ of $(1,0)$-forms satisfying

$$
d \omega^{1}=0, \quad d \omega^{2}=\omega^{1 \overline{1}} .
$$

A real $(1,1)$-form $F$ on $K T$ is closed if and only if

$$
F=\operatorname{ir} \omega^{1 \overline{1}}+u \omega^{1 \overline{2}}-\bar{u} \omega^{2 \overline{1}}
$$

for some $r \in \mathbb{R}$ and $u \in \mathbb{C}$. Since $F^{2}=-2|u|^{2} \omega^{12 \overline{1} \overline{2}}$, we have that $F$ is non-degenerate if and only if $u \neq 0$. Thus, there are pseudo-Kähler metrics on $K T$.

As a consequence of our previous discussion, we have:

Proposition 2.14. Let $X$ be a compact complex non-Kähler surface diffeomorphic to a solvmanifold. If $X$ admits a pseudo-Kähler metric, then $X$ is a primary Kodaira surface KT.

\section{Neutral Calabi-Yau manifolds}

In this section we focus our attention on a special kind of pseudo-Kähler manifolds, namely, neutral Calabi-Yau manifolds. Moreover, the intermediate class constituted by neutral Kähler manifolds is also studied. We first prove that compact neutral, Kähler or Calabi-Yau, surfaces are stable by small deformations of the complex structure. In higher dimensions, we construct an 8-dimensional nilmanifold endowed with a neutral Calabi-Yau metric that allows us to prove the instability of the neutral Kähler and neutral Calabi-Yau properties in any even complex dimension $n \geq 4$. It is worth to note that such nilmanifold also provides a counterexample to a conjecture in [13].

We first recall some definitions. Let $X=(M, J)$ be a complex manifold of complex dimension $n=2 m$. Following [20], a neutral Kähler structure on $X$ is a neutral metric $g$, i.e. of signature $(2 m, 2 m)$, such that

- $g$ is compatible with $J$, i.e. $g(J U, J V)=g(U, V)$ for any vector fields $U, V$ on $M$; and

- $J$ is parallel with respect to the Levi-Civita connection $\nabla$ of $g$, i.e. $\nabla J=0$. 
These conditions imply that the 2-form $F(U, V)=g(U, J V)$ is closed, i.e. a neutral Kähler structure is in particular pseudo-Kähler.

A neutral Kähler structure is said to be neutral Calabi-Yau if there exists a nowhere vanishing form $\Phi$ of bidegree $(2 m, 0)$ with respect to $J$ satisfying $\nabla \Phi=0$. Neutral Calabi-Yau manifolds are Ricci-flat.

3.1. Stability on compact complex surfaces. In this section we study the stability of the neutral Kähler and neutral Calabi-Yau properties in complex dimension 2.

Proposition 3.1. Let $X$ be a compact complex non-Kähler surface. Suppose that $X$ admits a neutral Kähler metric. Then, any sufficiently small deformation of $X$ also admits neutral Kähler metrics.

Proof. By Theorem 2.13, any sufficiently small deformation $X_{\mathbf{t}}$ of $X$ admits pseudo-Kähler metrics. Since $X$ is non-Kähler by hypothesis, the first Betti number of $X_{\mathbf{t}}$ is odd, so a pseudo-Kähler metric on $X_{\mathbf{t}}$ cannot have signature $(4,0)$ or $(0,4)$. Hence, the pseudo-Kähler metrics are necessarily neutral.

Let us now observe the following. On the one hand, Petean proves in [39, Proposition 5] that if a compact complex surface $X$ admits a Ricci-flat neutral Kähler metric, then its Kodaira dimension and its first Chern class are both zero. Moreover, $X$ must be a complex torus, a hyperelliptic surface, or a primary Kodaira surface [39, Corollary 2]. On the other hand, any compact complex surface with holomorphically trivial canonical bundle is isomorphic to a K3 surface, a torus, or a primary Kodaira surface. Hence, as a consequence of these results, one can ensure that the only compact complex surfaces that can admit neutral Calabi-Yau structures are a complex torus and a primary Kodaira surface. We know by [23. that these two are diffeomorphic to a 4-dimensional nilmanifold with an invariant complex structure. It is easy to check that they are both neutral Calabi-Yau. In fact, the result is clear for the torus, whereas for a primary Kodaira surface it suffices to remark that, according to the complex equations (7), the $(2,0)$-form $\Phi=\omega^{12}$ is nowhere vanishing and parallel with respect to the Levi-Civita connection of any neutral Kähler metric (8). Concerning deformations, it is well-known that the small deformations of the invariant complex structures on the complex torus or on the primary Kodaira surface are again invariant, so they admit neutral Calabi-Yau structures.

We sum up our previous discussion in the following result.

Proposition 3.2. Let $X$ be a compact neutral Calabi-Yau surface. Then, $X$ is a complex torus or a primary Kodaira surface. Moreover, any sufficiently small deformation of $X$ admits neutral Calabi-Yau structures.

Our next goal is to prove the instability of the neutral Calabi-Yau and neutral Kähler properties in complex dimension $n \geq 4$. We begin constructing an 8-dimensional nilmanifold endowed with neutral Calabi-Yau metrics. To our knowledge, this neutral Calabi-Yau nilmanifold is new and, in addition, it provides counterexamples to a conjecture on pseudo-Kähler nilpotent Lie algebras, as we will shortly see.

3.2. A neutral Calabi-Yau nilmanifold in eight dimensions. Let $M$ be a nilmanifold endowed with an invariant complex structure $J$. Let $n$ be the complex dimension of $X=(M, J)$, and suppose that $F$ is an invariant pseudo-Kähler metric on $X$. By [41], there always exists a closed (non-zero) invariant form $\Phi$ of bidegree $(n, 0)$ with respect to $J$, so $\nabla \Phi=0$ for the LeviCivita connection $\nabla$ of the invariant metric. Therefore, any invariant pseudo-Kähler metric $F$ on a complex nilmanifold $X$ is Ricci-flat (see [19]). 
Proposition 3.3. Let $X=(M, J)$ be the 8-dimensional nilmanifold $M$ endowed with the invariant complex structure $J$ defined by a basis of $(1,0)$-forms $\left\{\omega^{k}\right\}_{k=1}^{4}$ satisfying the structure equations

$$
\left\{\begin{aligned}
d \omega^{1} & =0 \\
d \omega^{2} & =-i \omega^{14}+i \omega^{1 \overline{4}} \\
d \omega^{3} & =\omega^{12}+\omega^{1 \overline{2}}-\omega^{2 \overline{1}} \\
d \omega^{4} & =-\omega^{1 \overline{3}}+\omega^{3 \overline{1}}
\end{aligned}\right.
$$

Then, $X$ admits pseudo-Kähler metrics. Moreover, any invariant pseudo-Kähler metric $F$ on $X$ is given by

$$
F=i\left(r \omega^{1 \overline{1}}+s \omega^{4 \overline{4}}\right)+u\left(\omega^{1 \overline{2}}-\omega^{2 \overline{1}}\right)+v\left(\omega^{1 \overline{3}}-\omega^{3 \overline{1}}\right)-s\left(\omega^{2 \overline{3}}-\omega^{3 \overline{2}}\right),
$$

where $r, s, u, v \in \mathbb{R}$ and $r s \neq 0$.

Proof. It is easy to check that the equations (9) satisfy the Jacobi identity, i.e. $d^{2} \omega^{k}=0$ for $1 \leq k \leq 4$. Thus, they define a simply-connected, connected, nilpotent Lie group $G$ of real dimension 8. Moreover, since the structure constants belong to $\mathbb{Q}[i]$, the well-known Malcev's theorem [33] ensures the existence of a lattice $\Gamma$ of maximal rank in $G$. We consider the nilmanifold $M=\Gamma \backslash G$, which is endowed by construction with the complex structure $J$ defined by the $(1,0)$ forms $\left\{\omega^{k}\right\}_{k=1}^{4}$.

Any invariant real 2 -form $F$ of bidegree $(1,1)$ on $X=(M, J)$ can be written as

$$
F=\sum_{k=1}^{4} i x_{k \bar{k}} \omega^{k \bar{k}}+\sum_{1 \leq k<l \leq 4}\left(x_{k \bar{l}} \omega^{k \bar{l}}-\bar{x}_{k \bar{l}} \omega^{l \bar{k}}\right),
$$

where $x_{k \bar{k}} \in \mathbb{R}$ and $x_{k \bar{l}} \in \mathbb{C}$, for $1 \leq k, l \leq 4$. Since we are looking for pseudo-Kähler metrics, we will study the condition $d F=0$ and the non-degeneracy condition $F^{4} \neq 0$.

By a direct computation using the complex structure equations (9) we get

$$
d F=\Theta+\bar{\Theta}
$$

where $\Theta=\partial F$ is the complex 3 -form of bidegree $(2,1)$ given by

$$
\begin{aligned}
\Theta= & 2 i \mathfrak{I m} x_{1 \overline{3}} \omega^{12 \overline{1}}+2 i \mathfrak{I m} x_{2 \overline{3}} \omega^{12 \overline{2}}-\left(x_{2 \overline{4}}-i x_{3 \overline{3}}\right) \omega^{12 \overline{3}}+x_{3 \overline{4}} \omega^{12 \overline{4}}-x_{1 \overline{4}} \omega^{13 \overline{1}}+i x_{3 \overline{3}} \omega^{13 \overline{2}} \\
& -x_{3 \overline{4}} \omega^{13 \overline{3}}+2 \mathfrak{I m} x_{1 \overline{2}} \omega^{14 \overline{1}}+\left(x_{2 \overline{2}}-\bar{x}_{3 \overline{4}}\right) \omega^{14 \overline{2}}-i\left(x_{2 \overline{3}}+x_{4 \overline{4}}\right) \omega^{14 \overline{3}}-i x_{2 \overline{4}} \omega^{14 \overline{4}} \\
& -\left(x_{2 \overline{4}}+i x_{3 \overline{3}}\right) \omega^{23 \overline{1}}+\left(x_{2 \overline{2}}+\bar{x}_{3 \overline{4}}\right) \omega^{24 \overline{1}}+i\left(x_{4 \overline{4}}+\bar{x}_{2 \overline{3}}\right) \omega^{34 \overline{1}} .
\end{aligned}
$$

Now, the closedness condition $d F=0$ is equivalent to $\Theta=0$. It is straightforward to check that the latter is satisfied if and only if

$$
x_{2 \overline{2}}=x_{3 \overline{3}}=x_{1 \overline{4}}=x_{2 \overline{4}}=x_{3 \overline{4}}=\mathfrak{I m} x_{1 \overline{2}}=\mathfrak{I m} x_{1 \overline{3}}=\mathfrak{I m} x_{2 \overline{3}}=0, \quad \text { and } \quad x_{2 \overline{3}}=-x_{4 \overline{4}} .
$$

Replacing these values in (11) and denoting $x_{1 \overline{1}}=r, x_{4 \overline{4}}=s, x_{1 \overline{2}}=u, x_{1 \overline{3}}=v$, with $r, s, u, v \in \mathbb{R}$, one directly gets the expression (10).

We finally need to ensure the non-degeneracy condition for $F$. Using (10), it is easy to see that

$$
F^{4}=-24 r s^{3} \omega^{1234 \overline{1} \overline{2} \overline{3} \overline{4}} .
$$

Therefore, $F^{4} \neq 0$ if and only if $r s \neq 0$, as stated in the proposition.

In the following result we prove that the family of pseudo-Kähler metrics (10) provides neutral Calabi-Yau metrics in eight dimensions that are not flat (although they all are Ricci flat). 
Proposition 3.4. The complex nilmanifold $X=(M, J)$ constructed in Proposition 3.3 has nonflat neutral Calabi-Yau structures.

Proof. Let us take the basis of real 1 -forms $\left\{e^{1}, \ldots, e^{8}\right\}$ on $X$ defined by $e^{2 k-1}+i e^{2 k}=\omega^{k}$, for $1 \leq k \leq 4$, where $\left\{\omega^{1}, \ldots, \omega^{4}\right\}$ is the basis of $(1,0)$-forms in Proposition 3.3 satisfying the complex structure equations (9). In terms of this real basis, the complex structure $J$ and the pseudo-Kähler metrics $F$ given in (10) express as

$$
\begin{aligned}
& J e^{1}=-e^{2}, \quad J e^{3}=-e^{4}, \quad J e^{5}=-e^{6}, \quad J e^{7}=-e^{8}, \\
& F=2 r e^{12}+2 u e^{13}+2 v e^{15}+2 u e^{24}+2 v e^{26}-2 s e^{35}-2 s e^{46}+2 s e^{78},
\end{aligned}
$$

where $r, s, u, v \in \mathbb{R}$ with $r s \neq 0$. The pseudo-Riemannian metric $g(x, y)=F(J x, y)$ is then given in terms of this real basis by the matrix

$$
\left(g_{i j}\right)_{i, j}=\left(\begin{array}{cccccccc}
2 r & 0 & 0 & -2 u & 0 & -2 v & 0 & 0 \\
0 & 2 r & 2 u & 0 & 2 v & 0 & 0 & 0 \\
0 & 2 u & 0 & 0 & 0 & 2 s & 0 & 0 \\
-2 u & 0 & 0 & 0 & -2 s & 0 & 0 & 0 \\
0 & 2 v & 0 & -2 s & 0 & 0 & 0 & 0 \\
-2 v & 0 & 2 s & 0 & 0 & 0 & 0 & 0 \\
0 & 0 & 0 & 0 & 0 & 0 & 2 s & 0 \\
0 & 0 & 0 & 0 & 0 & 0 & 0 & 2 s
\end{array}\right) .
$$

It is easy to see that there are metrics in the family (12) with neutral signature. In fact, taking for instance $u=v=0$, one has that $r s<0$ is equivalent to the signature being $(4,4)$. Since the $(4,0)$-form $\Phi=\omega^{1234}$ is parallel, we conclude that $X$ has neutral Calabi-Yau structures.

Let us now prove that any pseudo-Kähler metric (10) on $X$ is non-flat. Since the pseudo-Kähler structures are invariant, the (complexified) Koszul formula for the Levi-Civita connection $\nabla$ of the metric $g$ reduces to

$$
2 g\left(\nabla_{U} V, W\right)=g([U, V], W)-g([V, W], U)+g([W, U], V),
$$

for (invariant) complex vector fields $U, V, W$ on the complex nilmanifold $X$. Let $\left\{Z_{j}\right\}_{j=1}^{4}$ denote the basis of complex vector fields of bidegree $(1,0)$ dual to the basis $\left\{\omega^{j}\right\}_{j=1}^{4}$. Notice that, by complex conjugation, it suffices to compute $\nabla_{Z_{k}} Z_{j}$ and $\nabla_{\bar{Z}_{k}} Z_{j}$ for $1 \leq j, k \leq 4$. Furthermore, since $\nabla J=0$, one has that $\nabla_{U} V$ is of bidegree $(1,0)$ whenever $V$ is. In particular, $\nabla_{U} Z_{j}$ has type $(1,0)$ for every $1 \leq j \leq 4$.

Let $R$ be the curvature tensor of the pseudo-Kähler metric, i.e. $R$ is given by

$$
R(U, V, W, T)=g\left(\nabla_{U} \nabla_{V} W-\nabla_{V} \nabla_{U} W-\nabla_{[U, V]} W, T\right),
$$

for $U, V, W, T$ complex vector fields on $X$. Taking into account the observation in the previous paragraph, complex conjugation and the symmetries of the curvature tensor, one concludes that the metric $g$ is non-flat if and only if $R\left(Z_{i}, \bar{Z}_{j}, Z_{k}, \bar{Z}_{l}\right) \neq 0$ for some $i, j, k, l$. In our case, we will prove that $R\left(Z_{2}, \bar{Z}_{2}, Z_{2}, \bar{Z}_{2}\right) \neq 0$.

A direct calculation shows:

$$
\nabla_{\bar{Z}_{2}} Z_{1}=Z_{3}, \quad \nabla_{\bar{Z}_{2}} Z_{2}=0, \quad \nabla_{\bar{Z}_{2}} Z_{3}=0, \quad \text { and } \quad \nabla_{Z_{2}} Z_{2}=-\frac{i s}{r} Z_{1}-\frac{i v}{r} Z_{2}+\frac{i u}{r} Z_{3} .
$$


From the complex equations (9) we have $\left[Z_{2}, \bar{Z}_{2}\right]=0$. Hence,

$$
\begin{aligned}
R\left(Z_{2}, \bar{Z}_{2}, Z_{2}, \bar{Z}_{2}\right) & =g\left(\nabla_{Z_{2}} \nabla_{\bar{Z}_{2}} Z_{2}-\nabla_{\bar{Z}_{2}} \nabla_{Z_{2}} Z_{2}-\nabla_{\left[Z_{2}, \bar{Z}_{2}\right]} Z_{2}, \bar{Z}_{2}\right) \\
& =-g\left(\nabla_{\bar{Z}_{2}} \nabla_{Z_{2}} Z_{2}, \bar{Z}_{2}\right) \\
& =g\left(\frac{i s}{r} Z_{3}, \bar{Z}_{2}\right) \\
& =-\frac{s^{2}}{r} \neq 0 .
\end{aligned}
$$

Note that in 20] neutral Calabi-Yau structures on a specific class of nilmanifolds are constructed. This class is given by the so-called Kodaira manifolds, which are $4 m$-dimensional 2-step nilmanifolds whose underlying Lie algebras have center of dimension $2 \mathrm{~m}$. Moreover, their complex structure is invariant and preserves the center. Kodaira manifolds are a generalization of the Kodaira-Thurston manifold $K T$, and they have the structure of a principal torus bundle over a torus, with fiber the central torus.

It is worth to remark that, for the neutral Calabi-Yau nilmanifold $X=(M, J)$ constructed in Proposition 3.4, the center of the Lie algebra $\mathfrak{g}$ underlying $M$ is 1-dimensional. Hence, it is not invariant under the complex structure $J$ (see Section 3.2.1 for more details). Furthermore, $\mathfrak{g}$ has nilpotency step equal to 4. By [38], this implies that the neutral Calabi-Yau nilmanifold $X=(M, J)$ is far from being the total space of a principal torus bundle over a torus.

3.2.1. Counterexamples to a conjecture on pseudo-Kähler nilmanifolds. Here we show that the new pseudo-Kähler nilmanifold constructed in Proposition 3.3 provides counterexamples to a conjecture in [13. The conjecture states that an invariant complex structure $J$ on a nilmanifold $M=\Gamma \backslash G$ must satisfy a certain property so that $(M, J)$ admits pseudo-Kähler metrics. Let us first recall some results on complex structures on nilpotent Lie algebras (which can be found in [31] and the references therein) and then formulate the conjecture in precise terms.

Let $\mathfrak{g}$ be a nilpotent Lie algebra. Complex structures on $\mathfrak{g}$ can be classified into different types attending to the behaviour of the ascending $J$-compatible series of $\mathfrak{g}$, which is defined inductively as

$$
\mathfrak{a}_{0}(J)=\{0\}, \quad \mathfrak{a}_{k}(J)=\left\{X \in \mathfrak{g} \mid[X, \mathfrak{g}] \subseteq \mathfrak{a}_{k-1}(J) \text { and }[J X, \mathfrak{g}] \subseteq \mathfrak{a}_{k-1}(J)\right\}, \text { for } k \geq 1 .
$$

Note that $\mathfrak{a}_{k}(J)$ is an even-dimensional $J$-invariant ideal of $\mathfrak{g}$. In particular, $\mathfrak{a}_{1}(J)$ is the largest subspace of the center of $\mathfrak{g}$ which is $J$-invariant.

Unlike the usual ascending central series $\left\{\mathfrak{g}_{k}\right\}_{k}$ of $\mathfrak{g}$, the series $\left\{\mathfrak{a}_{k}(J)\right\}_{k}$ is adapted to the complex structure $J$, and it allows to introduce the following partition of the space of complex structures:

Definition 3.5. 31] A complex structure $J$ on a nilpotent Lie algebra $\mathfrak{g}$ is said to be

(i) nilpotent, if there exists an integer $t>0$ such that $\mathfrak{a}_{t}(J)=\mathfrak{g}$;

(ii) non-nilpotent, if $\mathfrak{a}_{t}(J) \neq \mathfrak{g}$ for every $t \geq 0$; moreover, $J$ is called

(ii.1) strongly non-nilpotent, if $\mathfrak{a}_{1}(J)=\{0\}$ (which implies $\mathfrak{a}_{t}(J)=\{0\}$ for every $t$ );

(ii.2) weakly non-nilpotent, if there is an integer $t>0$ satisfying $\{0\} \neq \mathfrak{a}_{t}(J)=\mathfrak{a}_{l}(J) \neq \mathfrak{g}$, for every $l \geq t$.

Notice that $\mathfrak{a}_{1}(J) \neq\{0\}$ for any nilpotent or weakly non-nilpotent complex structure $J$. This allows to construct such structures from other complex structures defined on lower dimensional nilpotent Lie algebras (see [31] for details). This fact leaves strongly non-nilpotent complex structures as the essentially new complex structures that arise in each even real dimension. In [31, Section 3.1] it is proved that if $\mathfrak{g}$ admits a strongly non-nilpotent complex structure $J$, then 
the nilpotency step of $\mathfrak{g}$ is at least 3 (see 31] for other general properties on Lie algebras with strongly non-nilpotent complex structures and structure results up to real dimension 8).

We can now formulate the following conjecture proposed in [13]:

Conjecture [13, page 123]: a complex structure on a 2n-dimensional nilpotent Lie algebra must be of nilpotent type in the presence of a compatible symplectic form.

It is proved in 13 that the conjecture holds for $n \leq 3$. However, the complex structure $J$ given in Proposition 3.3 provides a counterexample for $n=4$. In fact, from the equations (9) it is straightforward to prove that $\mathfrak{a}_{1}(J)=\{0\}$, that is to say, $J$ is strongly non-nilpotent according to Definition 3.5, and it admits the compatible symplectic forms given in (10). Furthermore, in every complex dimension $n \geq 4$ we have the following result.

Proposition 3.6. For each $n \geq 4$, there exists a $2 n$-dimensional nilmanifold endowed with a non-nilpotent complex structure that admits pseudo-Kähler metrics.

Proof. Let us consider $Y=X \times \mathbb{T}^{k}$, where $X=(M, J)$ is the 8-dimensional pseudo-Kähler nilmanifold given in Proposition 3.3 and $\mathbb{T}^{k}$ denotes the $k$-dimensional complex torus endowed with any invariant pseudo-Kähler metric. Then, $Y$ is a pseudo-Kähler nilmanifold of real dimension $2 n=8+2 k$ with invariant complex structure $J_{Y}=J \times J_{\mathbb{T}^{k}}$ satisfying $\{0\} \neq \mathfrak{b}=\mathfrak{a}_{t}\left(J_{Y}\right) \neq \mathfrak{g} \times \mathfrak{b}$, for every $t>0$, where $\mathfrak{b}$ denotes the abelian Lie algebra underlying the torus $\mathbb{T}^{k}$, and $\mathfrak{g}$ the Lie algebra of $M$. In particular, $J_{Y}$ is (weakly) non-nilpotent.

3.3. Instability in complex dimension $n \geq 4$. In contrast to the stability results for compact complex surfaces proved in Section 3.1, we will next show that the neutral Kähler and neutral Calabi-Yau properties are both unstable in every complex dimension $n \geq 4$. This constitutes a deep difference with the Kähler Calabi-Yau case, for which the deformation space is unobstructed by the well-known Bogomolov-Tian-Todorov theorem [8, 47, 48]. We begin with the following result in complex dimension 4.

Theorem 3.7. There exists a holomorphic family of compact complex manifolds $\left\{X_{\mathbf{t}}\right\}_{\mathbf{t} \in \Delta}$ of complex dimension 4 , where $\Delta=\{\mathbf{t} \in \mathbb{C}|| \mathbf{t} \mid<1\}$, satisfying the following properties:

(i) $X_{0}$ is a neutral Calabi-Yau manifold;

(ii) $X_{\mathbf{t}}$ does not admit any pseudo-Kähler structure for $\mathbf{t} \in \Delta \backslash \mathcal{C}$, where $\mathcal{C}$ is the real curve through 0 given by $\mathcal{C}=\{\mathbf{t} \in \Delta \mid$ Re $\mathbf{t}=0\}$.

Therefore, neither the neutral Calabi-Yau property nor the neutral Kähler property are stable under deformations of the complex structure.

Proof. The proof is based on an appropriate deformation of the neutral Calabi-Yau nilmanifold $X=(M, J)$ found in Proposition 3.4.

Let $\left\{\omega^{k}\right\}_{k=1}^{4}$ be the basis of $(1,0)$-forms on $X$ satisfying (9). Observe that the $(0,1)$-form $\omega^{\overline{1}}$ defines a Dolbeault cohomology class on $X$. We consider the class $\left[\omega^{\overline{1}}\right] \in H_{\bar{\partial}}^{0,1}(X)$ to perform an appropriate holomorphic deformation of $X$. For each $\mathbf{t} \in \mathbb{C}$ such that $|\mathbf{t}|<1$, we define the complex structure $J_{\mathbf{t}}$ on $M$ given by the following basis $\left\{\eta_{\mathbf{t}}^{k}\right\}_{k=1}^{4}$ of $(1,0)$-forms:

$$
\eta_{\mathbf{t}}^{1}:=\omega^{1}, \quad \eta_{\mathbf{t}}^{2}:=\omega^{2}-\mathbf{t} \omega^{\overline{1}}, \quad \eta_{\mathbf{t}}^{3}:=\omega^{3}, \quad \eta_{\mathbf{t}}^{4}:=\omega^{4} .
$$


The complex structure equations for $X_{\mathbf{t}}=\left(M, J_{\mathbf{t}}\right)$ are

$$
\left\{\begin{array}{l}
d \eta_{\mathbf{t}}^{1}=0 \\
d \eta_{\mathbf{t}}^{2}=-i \eta_{\mathbf{t}}^{14}+i \eta_{\mathbf{t}}^{1 \overline{4}} \\
d \eta_{\mathbf{t}}^{3}=\eta_{\mathbf{t}}^{12}+\mathbf{t} \eta_{\mathbf{t}}^{1 \overline{1}}+\eta_{\mathbf{t}}^{1 \overline{2}}-\eta_{\mathbf{t}}^{2 \overline{1}} \\
d \eta_{\mathbf{t}}^{4}=-\eta_{\mathbf{t}}^{1 \overline{3}}+\eta_{\mathbf{t}}^{3 \overline{1}}
\end{array}\right.
$$

Observe that the initial structure $J$ is recovered for $\mathbf{t}=0$. Therefore, $X_{0}=X$ and one immediately gets part (i) of the statement.

To prove (ii), since $X_{\mathbf{t}}$ is a complex nilmanifold, a similar argument as in the proof of Proposition 2.1 allows us to reduce the study of existence of pseudo-Kähler metrics on $X_{\mathbf{t}}$ to the study of invariant ones. We first analyze the existence of invariant closed 2 -forms $\Omega$ on $M$ compatible with $J_{\mathbf{t}}$, not necessarily real. Any such $\Omega$ belongs to $\bigwedge_{J_{\mathbf{t}}}^{1,1}\left(\mathfrak{g}^{*}\right)$, where $\mathfrak{g}$ denotes the Lie algebra underlying the nilmanifold $M$, and it is given by

$$
\Omega=\sum_{k=1}^{4}\left(a_{k} \eta_{\mathbf{t}}^{1}+b_{k} \eta_{\mathbf{t}}^{2}+c_{k} \eta_{\mathbf{t}}^{3}+f_{k} \eta_{\mathbf{t}}^{4}\right) \wedge \eta_{\mathbf{t}}^{\bar{k}},
$$

where $a_{k}, b_{k}, c_{k}, f_{k} \in \mathbb{C}$, for $1 \leq k \leq 4$. Making use of the complex structure equations (14), one has

where

$$
d \Omega=\partial_{\mathbf{t}} \Omega+\bar{\partial}_{\mathbf{t}} \Omega
$$

$$
\begin{aligned}
\partial_{\mathbf{t}} \Omega= & \left(a_{3}-\overline{\mathbf{t}} b_{3}+c_{1}\right) \eta_{\mathbf{t}}^{12 \overline{1}}+\left(b_{3}+c_{2}\right) \eta_{\mathbf{t}}^{12 \overline{2}}+\left(c_{3}-b_{4}\right) \eta_{\mathbf{t}}^{12 \overline{3}}+c_{4} \eta_{\mathbf{t}}^{12 \overline{4}}-\left(a_{4}+\overline{\mathbf{t}} c_{3}\right) \eta_{\mathbf{t}}^{13 \overline{1}} \\
& +c_{3} \eta_{\mathbf{t}}^{13 \overline{2}}-c_{4} \eta_{\mathbf{t}}^{13 \overline{3}}-i\left(a_{2}+b_{1}-i \overline{\mathbf{t}} f_{3}\right) \eta_{\mathbf{t}}^{14 \overline{1}}+\left(f_{3}-i b_{2}\right) \eta_{\mathbf{t}}^{14 \overline{2}}-\left(f_{4}+i b_{3}\right) \eta_{\mathbf{t}}^{14 \overline{3}} \\
& -i b_{4} \eta_{\mathbf{t}}^{14 \overline{4}}-\left(b_{4}+c_{3}\right) \eta_{\mathbf{t}}^{23 \overline{1}}-\left(f_{3}+i b_{2}\right) \eta_{\mathbf{t}}^{24 \overline{1}}+\left(f_{4}-i c_{2}\right) \eta_{\mathbf{t}}^{34 \overline{1}}
\end{aligned}
$$

and

$$
\begin{aligned}
\bar{\partial}_{\mathbf{t}} \Omega= & -\left(a_{3}+c_{1}-\mathbf{t} c_{2}\right) \eta_{\mathbf{t}}^{1 \overline{1} \overline{2}}+\left(f_{1}+\mathbf{t} c_{3}\right) \eta_{\mathbf{t}}^{1 \overline{1} \overline{3}}-i\left(a_{2}+b_{1}+i \mathbf{t} c_{4}\right) \eta_{\mathbf{t}}^{1 \overline{1} \overline{4}}+\left(c_{3}+f_{2}\right) \eta_{\mathbf{t}}^{1 \overline{2} \overline{3}} \\
& +\left(c_{4}-i b_{2}\right) \eta_{\mathbf{t}}^{1 \overline{2} \overline{4}}-\left(f_{4}+i b_{3}\right) \eta_{\mathbf{t}}^{1 \overline{3} \overline{4}}-\left(b_{3}+c_{2}\right) \eta_{\mathbf{t}}^{2 \overline{1} \overline{2}}-c_{3} \eta_{\mathbf{t}}^{2 \overline{1} \overline{3}}-\left(c_{4}+i b_{2}\right) \eta_{\mathbf{t}}^{2 \overline{1} \overline{4}} \\
& +\left(f_{2}-c_{3}\right) \eta_{\mathbf{t}}^{3 \overline{1} \overline{2}}+f_{3} \eta_{\mathbf{t}}^{3 \overline{1} \overline{3}}+\left(f_{4}-i c_{2}\right) \eta_{\mathbf{t}}^{3 \overline{4} \overline{4}}-f_{3} \eta_{\mathbf{t}}^{4 \overline{1} \overline{2}}-i f_{2} \eta_{\mathbf{t}}^{4 \overline{1} \overline{4}} .
\end{aligned}
$$

From these expressions we see that $\Omega$ is closed, i.e. $\partial_{\mathbf{t}} \Omega=0=\bar{\partial}_{\mathbf{t}} \Omega$, if and only if

$$
a_{4}=b_{2}=b_{4}=c_{3}=c_{4}=f_{1}=f_{2}=f_{3}=0,
$$

and

$$
b_{1}=-a_{2}, \quad c_{2}=-b_{3}, \quad f_{4}=-i b_{3}, \quad-a_{3}+\overline{\mathbf{t}} b_{3}=c_{1}=-a_{3}-\mathbf{t} b_{3} .
$$

Now, the latter two equalities imply the equation

$$
b_{3} \mathfrak{R e} \mathbf{t}=0,
$$

which gives rise to the following two cases.

If $\mathfrak{R e} \mathbf{t}=0$, then pseudo-Kähler metrics exist on $X_{\mathbf{t}}$; for instance

$$
F=i \eta_{\mathbf{t}}^{1 \overline{1}}-i \eta_{\mathbf{t}}^{4 \overline{4}}-\frac{\mathbf{t}}{2} \eta_{\mathbf{t}}^{1 \overline{3}}+\eta_{\mathbf{t}}^{2 \overline{3}}+\frac{\overline{\mathbf{t}}}{2} \eta_{\mathbf{t}}^{3 \overline{1}}-\eta_{\mathbf{t}}^{3 \overline{2}}
$$

is a real closed $(1,1)$-form which is non-degenerate.

However, if we assume $\mathfrak{R e} \mathbf{t} \neq 0$, then one has $b_{3}=0$ and consequently $c_{2}=f_{4}=0$. Thus, every closed 2 -form $\Omega$ of bidegree $(1,1)$ with respect to the complex structure $J_{\mathbf{t}}$ is given by

$$
\Omega=a_{1} \eta_{\mathbf{t}}^{1 \overline{1}}+a_{2}\left(\eta_{\mathbf{t}}^{1 \overline{2}}-\eta_{\mathbf{t}}^{2 \overline{1}}\right)+a_{3}\left(\eta_{\mathbf{t}}^{1 \overline{3}}-\eta_{\mathbf{t}}^{3 \overline{1}}\right),
$$


with $a_{1}, a_{2}, a_{3} \in \mathbb{C}$.

Hence, when $\mathfrak{R e} \mathbf{t} \neq 0$, the space of real closed 2 -forms on $\mathfrak{g}$ compatible with $J_{\mathbf{t}}$, namely $\mathcal{Z}_{J_{\mathbf{t}}}^{+}(\mathfrak{g})=\left\{\alpha \in \wedge^{2} \mathfrak{g}^{*} \mid d \alpha=0\right.$ and $\left.J_{\mathbf{t}} \alpha=\alpha\right\}=\left\{\alpha \in \wedge_{J_{\mathbf{t}}}^{1,1} \mathfrak{g}_{\mathbb{C}}^{*} \mid d \alpha=0\right.$ and $\left.\bar{\alpha}=\alpha\right\}$, is generated by

$$
\mathcal{Z}_{J_{\mathbf{t}}}^{+}(\mathfrak{g})=\left\langle i \eta_{\mathbf{t}}^{1 \overline{1}}, \eta_{\mathbf{t}}^{1 \overline{2}}-\eta_{\mathbf{t}}^{2 \overline{1}}, \eta_{\mathbf{t}}^{1 \overline{3}}-\eta_{\mathbf{t}}^{3 \overline{1}}\right\rangle .
$$

Since every element in $\mathcal{Z}_{J_{\mathbf{t}}}^{+}(\mathfrak{g})$ is degenerate, no pseudo-Kähler metrics exist on $X_{\mathbf{t}}=\left(M, J_{\mathbf{t}}\right)$ when $\mathfrak{R e} \mathbf{t} \neq 0$. This clearly implies that $X_{\mathbf{t}}$ cannot admit any neutral Kähler or neutral Calabi-Yau structure for $\mathbf{t} \in \Delta \backslash \mathcal{C}$, where $\mathcal{C}=\{\mathbf{t} \in \Delta \mid \mathfrak{R e} \mathbf{t}=0\}$. Hence, both properties are unstable by small deformations.

In the following theorem we sum up the main results about instability found along the preceding sections.

Theorem 3.8. On compact complex manifolds of complex dimension $\geq 3$, the properties of "being pseudo-Kähler", "being neutral Kähler" and "being neutral Calabi-Yau" are not stable under small deformations of the complex structure.

Proof. For the pseudo-Kähler property, the result follows from Proposition 2.1 and Remark 2.2 , For the neutral Kähler and neutral Calabi-Yau properties in complex dimension 4, the result is given in Theorem 3.7. In higher dimensions, it suffices to consider the product $Y_{\mathbf{t}}=X_{\mathbf{t}} \times \mathbb{T}^{2 m}$, where $X_{\mathbf{t}}=\left(M, J_{\mathbf{t}}\right)$ is the holomorphic family given in Theorem 3.7 and $\mathbb{T}^{2 m}$ the $2 m$-dimensional complex torus endowed with an invariant neutral Calabi-Yau metric. If $\mathfrak{g}$ and $\mathfrak{b}$ denote the Lie algebras underlying $M$ and $\mathbb{T}^{2 m}$, respectively, then the space of invariant closed real 2-forms on $Y_{\mathbf{t}}$ compatible with the product complex structure $J_{Y_{\mathbf{t}}}=J_{\mathbf{t}} \times J_{\mathbb{T}^{2 m}}$ is given by

$$
\mathcal{Z}_{J_{Y_{\mathbf{t}}}}^{+}(\mathfrak{g} \times \mathfrak{b})=\mathcal{Z}_{J_{\mathfrak{t}}}^{+}(\mathfrak{g}) \oplus\left\{\eta_{\mathbf{t}}^{1} \wedge \bar{\alpha}-\alpha \wedge \eta_{\mathbf{t}}^{\overline{1}} \mid \alpha \in \Lambda_{J_{\mathbb{T}^{2 m}}}^{1,0} \mathfrak{b}^{*}\right\} \oplus \mathcal{Z}_{J_{\mathbb{T}^{2 m}}^{+}}(\mathfrak{b}),
$$

where the space $\mathcal{Z}_{J_{\mathbf{t}}}^{+}(\mathfrak{g})$ is described in (15) for any $\mathbf{t} \in \Delta$ such that $\mathfrak{R e} \mathbf{t} \neq 0$. It is easy to see that any element in $\mathcal{Z}_{J_{Y_{\mathbf{t}}}}^{+}(\mathfrak{g} \times \mathfrak{b})$ is degenerate, so $Y_{\mathbf{t}}$ does not admit pseudo-Kähler metrics for any $\mathbf{t} \in \Delta \backslash \mathcal{C}$. Since $Y_{0}=X_{0} \times \mathbb{T}^{2 m}$ is neutral Calabi-Yau, the result follows immediately.

\section{Pseudo-Hermitian-Symplectic structures}

In this section we consider an indefinite version of the Hermitian-symplectic geometry. The motivation comes from the fact that the small deformations of any pseudo-Kähler manifold always posses what we will call a pseudo-Hermitian-symplectic structure. We will show that there are compact complex manifolds with pseudo-Hermitian-symplectic structure but not admitting any pseudo-Kähler metric.

Recall that a complex structure $J$ on a symplectic manifold $(M, \Omega)$ is said to be tamed by the symplectic form $\Omega$ if the condition $\Omega(x, J x)>0$ is satisfied for all non-zero tangent vectors $x$. Following the terminology of [44], we will refer to the pair $(\Omega, J)$ as a Hermitian-symplectic structure. Note that the tamed condition is equivalent to require that the $(1,1)$-component $\Omega^{1,1}$ of the symplectic form $\Omega$ is positive, i.e. $\Omega^{1,1}$ is a Hermitian metric on the complex manifold $X=(M, J)$. No example of a non-Kähler compact complex manifold admitting a Hermitiansymplectic structure is known (see [32, page 678] and [44, Question 1.7]).

By analogy, in the pseudo-Hermitian setting, we introduce the following notion:

Definition 4.1. A complex manifold $X=(M, J)$ is called pseudo-Hermitian-symplectic if there exists a symplectic form $\Omega$ on $M$ such that its component of bidegree (1,1) with respect to $J$ is non-degenerate. In such case we will say that the pair $(\Omega, J)$ is a pseudo-Hermitian-symplectic structure. 
From the definition, it is clear that any pseudo-Kähler manifold is pseudo-Hermitian-symplectic.

Example 4.2. For a primary Kodaira surface, with equations given by (7), the pseudo-Hermitiansymplectic (indeed pseudo-Kähler) structures are defined in (8). Observe that the form $\Omega=$ $\omega^{12}+\omega^{\overline{1} 2}$ is symplectic, but the pair $(\Omega, J)$ is not pseudo-Hermitian-symplectic because the $(1,1)$ component of $\Omega$ is identically zero.

Remark 4.3. In the positive-definite case, [17, Proposition 2.1] provides an important characterization of the Hermitian-symplectic condition. More precisely, the existence of such a structure on a complex manifold $X$ is shown to be equivalent to the existence of a Hermitian metric $F$ satisfying $\partial F=\bar{\partial} \alpha$, for some $\partial$-closed $(2,0)$-form $\alpha$ on $X$. In the following example we illustrate that a similar result does not hold in the pseudo-Hermitian-symplectic setting.

Example 4.4. Let us consider a compact complex nilmanifold defined by the complex equations

$$
d \omega^{1}=d \omega^{2}=0, \quad d \omega^{3}=\rho \omega^{12}+\omega^{1 \overline{1}}+\rho \omega^{1 \overline{2}}+D \omega^{2 \overline{2}},
$$

for some $D \in \mathbb{C}$ and $\rho \in\{0,1\}$.

Let $F=\frac{i}{2} r \omega^{1 \overline{1}}-\omega^{2 \overline{3}}+\omega^{3 \overline{2}}$, where $r \in \mathbb{R}^{*}$. Then, $F$ is a real form of bidegree $(1,1)$ and $F^{3}=3 \operatorname{ir} \omega^{1 \overline{1} 2 \overline{2} 3 \overline{3}} \neq 0$. Moreover, the $(2,0)$-form $\alpha=-\omega^{23}$ is $\partial$-closed and satisfies

$$
\partial F=\omega^{12 \overline{1}}+\rho \omega^{12 \overline{2}}=-\bar{\partial} \alpha .
$$

Take now the real 2-form $\Omega=\alpha+F+\bar{\alpha}$. By the previous condition we have $\partial \alpha=\bar{\partial} \alpha+\partial F=0$, which implies $d \Omega=0$.

However, the closed 2 -form $\Omega$ is not symplectic. In fact,

$$
\Omega^{3}=(\alpha+F+\bar{\alpha})^{3}=F^{3}+6 \alpha \wedge \bar{\alpha} \wedge F=0 .
$$

Even more, if $\rho=0$ and $D \in \mathbb{R}^{*}$, then the corresponding nilmanifold does not admit any symplectic structure (indeed, the underlying Lie algebra is $\mathfrak{h}_{3}$ in the notation of [49]).

For the other values of $\rho$ and $D$ we have by [11, Proposition 2.4] that the nilmanifold has underlying Lie algebra $\mathfrak{h}_{2}, \mathfrak{h}_{4}, \mathfrak{h}_{6}$ or $\mathfrak{h}_{8}$. These nilmanifolds are symplectic.

We next prove that, similarly to the Hermitian case [51, Proposition 2.4], the pseudo-Hermitiansymplectic property is open under holomorphic deformations.

Proposition 4.5. For compact complex manifolds, the pseudo-Hermitian-symplectic property is stable. Therefore, any sufficiently small deformation of a pseudo-Kähler manifold is pseudoHermitian-symplectic.

Proof. Let us denote by $M$ the real manifold underlying a complex manifold $X$ and by $J$ the complex structure on $M$ such that $X=(M, J)$. Let $\Omega$ be a symplectic form on $M$ whose $(1,1)$ component with respect to $J$ is non-degenerate, i.e. $(\Omega, J)$ is a pseudo-Hermitian-symplectic structure. We consider a holomorphic family of compact complex manifolds $\left\{X_{\mathbf{t}}=\left(M, J_{\mathbf{t}}\right)\right\}_{\mathbf{t} \in \Delta}$, with $\Delta$ containing 0 , such that $X_{0}=X$.

The symplectic form $\Omega$ decomposes on the compact complex manifold $X_{\mathbf{t}}$ as

$$
\Omega=\alpha_{\mathbf{t}}+F_{\mathbf{t}}+\beta_{\mathbf{t}}
$$

where $\alpha_{\mathbf{t}}$ has bidegree $(2,0), F_{\mathbf{t}}$ is the $(1,1)$ component of $\Omega$, and $\beta_{\mathbf{t}}=\bar{\alpha}_{\mathbf{t}}$. By hypothesis, for $\mathbf{t}=0$ the form $F_{0}$ is non-degenerate. Hence, one concludes that $F_{\mathbf{t}}$ is also non-degenerate for any $\mathbf{t} \in \Delta$ sufficiently close to $0 \in \Delta$, so $X_{\mathbf{t}}$ satisfies the pseudo-Hermitian-symplectic property.

The second assertion in the proposition is clear since any pseudo-Kähler manifold is pseudoHermitian-symplectic. 
As we recalled above, Streets and Tian pose in [44, Question 1.7] the following question, which is still an open problem: Does there exist a compact complex manifold, of complex dimension $\geq 3$, admitting a Hermitian-symplectic structure but no Kähler metrics?

In the following result we prove that the indefinite counterpart of this problem has an affirmative answer.

Proposition 4.6. There exist compact complex manifolds with pseudo-Hermitian-symplectic structure but not admitting any pseudo-Kähler metric.

Proof. Let us consider the family of compact complex manifolds $\left\{X_{\mathbf{t}}\right\}_{\mathbf{t} \in \Delta}$, of complex dimension 3 , constructed in Proposition 2.1. We have:

(i) $X_{0}$ is a pseudo-Kähler manifold,

(ii) $X_{\mathbf{t}}$ does not admit any pseudo-Kähler metric for $\mathbf{t} \neq 0$.

Since $X_{0}$ is a pseudo-Kähler manifold, by Proposition 4.5 and (ii) we conclude that, for sufficiently small values of $\mathbf{t} \neq 0$, the compact complex manifold $X_{\mathbf{t}}$ is a pseudo-Hermitian-symplectic manifold with no pseudo-Kähler metrics.

In the next examples we consider pseudo-Kähler structures on a compact complex manifold $X_{0}$ and illustrate their behaviour along a small holomorphic deformation $X_{\mathbf{t}}$ of $X_{0}$.

Example 4.7. Let us consider the family of compact complex manifolds $\left\{X_{\mathbf{t}}\right\}_{\mathbf{t} \in \Delta=\{\mathbf{t} \in \mathbb{C}|| \mathbf{t} \mid<1\}}$ of complex dimension 3 constructed in Proposition 2.1. Using the complex equations (11) of the complex nilmanifold $X_{0}$, we have that any invariant pseudo-Kähler metric $F$ on $X_{0}$ is given by

$$
F=i\left(r \omega^{1 \overline{1}}+s \omega^{2 \overline{2}}\right)+u \omega^{1 \overline{2}}-\bar{u} \omega^{2 \overline{1}}+v \omega^{2 \overline{3}}-\bar{v} \omega^{3 \overline{2}}
$$

for some $r, s \in \mathbb{R}$ and $u, v \in \mathbb{C}$ satisfying $r v \neq 0$.

A direct calculation using (2) shows that the 2-form $F$ decomposes along the deformation $X_{\mathbf{t}}$ as $F=\alpha_{\mathbf{t}}+F_{\mathbf{t}}+\beta_{\mathbf{t}}=-\frac{v \overline{\mathbf{t}}}{1-|\mathbf{t}|^{2}} \omega_{\mathbf{t}}^{23}+i\left(r \omega_{\mathbf{t}}^{1 \overline{1}}+s \omega_{\mathbf{t}}^{2 \overline{2}}\right)+u \omega_{\mathbf{t}}^{1 \overline{2}}-\bar{u} \omega_{\mathbf{t}}^{2 \overline{1}}+\frac{1}{1-|\mathbf{t}|^{2}}\left(v \omega_{\mathbf{t}}^{2 \overline{3}}-\bar{v} \omega_{\mathbf{t}}^{3 \overline{2}}\right)-\frac{\bar{v} \mathbf{t}}{1-|\mathbf{t}|^{2}} \omega_{\mathbf{t}}^{\overline{2} \overline{3}}$, where $\alpha_{\mathbf{t}}=-\frac{v \overline{\mathbf{t}}}{1-|\mathbf{t}|^{2}} \omega_{\mathbf{t}}^{23}$ is the $(2,0)$-component of $F$, and $\beta_{\mathbf{t}}=\bar{\alpha}_{\mathbf{t}}$. The real $(1,1)$-form $F_{\mathbf{t}}$ is non-degenerate on the manifold $X_{\mathbf{t}}$, i.e. $F$ defines a pseudo-Hermitian-symplectic structure on $X_{\mathbf{t}}$, which is in accord to Proposition 4.5. Notice that $d F_{\mathbf{t}}=\frac{1}{1-|\mathbf{t}|^{2}}\left(v \overline{\mathbf{t}} \omega_{\mathbf{t}}^{12 \overline{2}}+\bar{v} \mathbf{t} \omega_{\mathbf{t}}^{2 \overline{1} \overline{2}}\right) \neq 0$ for any $\mathbf{t} \in \Delta \backslash\{0\}$, i.e. the (1,1)-form $F_{\mathbf{t}}$ is not closed. Furthermore, by Proposition 2.1 (ii), $X_{\mathbf{t}}$ does not admit any pseudo-Kähler structure for $\mathbf{t} \in \Delta \backslash\{0\}$.

Example 4.8. Let $\left\{X_{\mathbf{t}}\right\}_{\mathbf{t} \in \Delta=\{\mathbf{t} \in \mathbb{C}|| \mathbf{t} \mid<1\}}$ be the family of compact complex manifolds of complex dimension 4 constructed in Theorem [3.7. The manifold $X_{0}$ is neutral Calabi-Yau, hence pseudoKähler, and by (10) any invariant pseudo-Kähler metric $F$ on $X_{0}$ is given by

$$
F=i\left(r \omega^{1 \overline{1}}+s \omega^{4 \overline{4}}\right)+u\left(\omega^{1 \overline{2}}-\omega^{2 \overline{1}}\right)+v\left(\omega^{1 \overline{3}}-\omega^{3 \overline{1}}\right)-s\left(\omega^{2 \overline{3}}-\omega^{3 \overline{2}}\right),
$$

for some $r, s, u, v \in \mathbb{R}$ with $r s \neq 0$. A direct calculation using (13) shows that the 2-form $F$ decomposes as follows along the deformation $X_{\mathbf{t}}$ :

$F=\alpha_{\mathbf{t}}+F_{\mathbf{t}}+\beta_{\mathbf{t}}=-s \overline{\mathbf{t}} \eta_{\mathbf{t}}^{13}+i\left(r \eta_{\mathbf{t}}^{1 \overline{1}}+s \eta_{\mathbf{t}}^{4 \overline{4}}\right)+u\left(\eta_{\mathbf{t}}^{1 \overline{2}}-\eta_{\mathbf{t}}^{2 \overline{1}}\right)+v\left(\eta_{\mathbf{t}}^{1 \overline{3}}-\eta_{\mathbf{t}}^{3 \overline{1}}\right)-s\left(\eta_{\mathbf{t}}^{2 \overline{3}}-\eta_{\mathbf{t}}^{3 \overline{2}}\right)-s \mathbf{t} \eta_{\mathbf{t}}^{\overline{1} \overline{3}}$, where $\alpha_{\mathbf{t}}=-s \overline{\mathbf{t}} \eta_{\mathbf{t}}^{13}$ is the (2,0)-component of the form $F$, and $\beta_{\mathbf{t}}=\bar{\alpha}_{\mathbf{t}}$. The real (1,1)-form $F_{\mathbf{t}}$ defines a pseudo-Hermitian-symplectic structure on $X_{\mathbf{t}}$ as it is non-degenerate, accordingly to Proposition 4.5. Note that $F_{\mathbf{t}}$ is not closed for any $\mathbf{t} \in \Delta \backslash\{0\}$, since $d F_{\mathbf{t}}=s\left(\overline{\mathbf{t}} \eta_{\mathbf{t}}^{12 \overline{1}}+\mathbf{t} \eta_{\mathbf{t}}^{1 \overline{1} \overline{2}}\right)$. Moreover, by Theorem 3.7 (ii), the compact complex manifold $X_{\mathbf{t}}$ does not admit any pseudoKähler structure for every $\mathbf{t} \in \Delta \backslash \mathcal{C}$, where $\mathcal{C}=\{\mathbf{t} \in \Delta \mid \mathfrak{R e} \mathbf{t}=0\}$. 


\section{ACKNOWLEDGMENTS}

This work has been partially supported by the projects MTM2017-85649-P (AEI/FEDER, UE), and E22-17R "Algebra y Geometría” (Gobierno de Aragón/FEDER).

\section{REFERENCES}

[1] A. Aeppli, On the cohomology structure of Stein manifolds, Proc. Conf. Complex Analysis (Minneapolis, Minn., 1964), Springer, Berlin (1965), 58-70.

[2] L.C. de Andrés, M. Fernández, A. Gray, J.J. Mencía, Compact manifolds with indefinite Kähler metrics, Proceedings of the Sixth International Colloquium on Differential Geometry (Santiago de Compostela, 1988), Cursos Congr. Univ. Santiago de Compostela 61 (1989), 25-50.

[3] D. Angella, Cohomological aspects in complex non-Kähler geometry, Lecture Notes in Math. 2095, Springer 2014.

[4] D. Angella, M.G. Franzini, F.A. Rossi, Degree of non-Kählerianity for 6-dimensional nilmanifolds, Manuscripta Math. 148 (2015), no. 1-2, 177-211.

[5] D. Angella, H. Kasuya, Cohomologies of deformations of solvmanifolds and closedness of some properties, North-West. Eur. J. Math. 3 (2017), 75-105, i.

[6] D. Angella, A. Tomassini, On the $\partial \bar{\partial}$-Lemma and Bott-Chern cohomology, Invent. Math. 192 (2013), 71-81.

[7] F. A. Belgun, On the metric structure of non-Kähler complex surfaces, Math. Ann. 317 (2000), no. 1, 1-40.

[8] F.A. Bogomolov, Hamiltonian Kähler manifolds, Dolk. Akad. Nauk SSSR 243 (1978), 1101-1104.

[9] R. Bott, S.-S. Chern, Hermitian vector bundles and the equidistribution of the zeroes of their holomorphic sections, Acta Math. 114 (1965), 71-112.

[10] N. Buchdahl, On compact Kähler surfaces, Ann. Inst. Fourier 49 (1999), 287-302.

[11] M. Ceballos, A. Otal, L. Ugarte, R. Villacampa, Invariant complex structures on 6-nilmanifolds: classification, Frölicher spectral sequence and special Hermitian metrics, J. Geom. Anal. 26 (2016), no. 1, 252-286.

[12] L.A. Cordero, M. Fernández, L. Ugarte, Lefschetz complex conditions for complex manifolds, Ann. Global Anal. Geom. 22 (2002), 355-373.

[13] L.A. Cordero, M. Fernández, L. Ugarte, Pseudo-Kähler metrics on six-dimensional nilpotent Lie algebras, J. Geom. Phys. 50 (2004), 115-137.

[14] A. Dancer, A. Swann, Hypersymplectic manifolds, In: Recent Developments in Pseudo-Riemannian Geometry. ESI Lect. Math. Phys., EMS, Zurich, 97-111, 2008.

[15] P. Deligne, P. Griffiths, J. Morgan, D. Sullivan, Real homotopy theory of Kähler manifolds, Invent. Math. 29 (1975), 245-274.

[16] J. Dorfmeister, Z.-D. Guan, Classification of compact homogeneous pseudo-Kähler manifolds, Comment. Math. Helv. 67 (1992), 499-513.

[17] N. Enrietti, A. Fino, L. Vezzoni, Tamed symplectic forms and SKT metrics, J. Symplectic Geom. 10 (2012), 203-224.

[18] C. Ehresmann, Sur les espaces fibrés différentiables, C. R. Acad. Sci. Paris 224 (1947), 1611-1612.

[19] A. Fino, M. Parton, S. Salamon, Families of strong KT structures in six dimensions, Comment. Math. Helv. 79 (2004), 317-340.

[20] A. Fino, H. Pedersen, Y.S. Poon, M.W. Sørensen, Neutral Calabi-Yau structures on Kodaira manifolds, Commun. Math. Phys. 248 (2004), 255-268.

[21] D. Guan, Classification of compact complex homogeneous manifolds with pseudo-Kählerian structures, J. Algebra 324 (2010), 2010-2024.

[22] K. Hasegawa, Small deformations and non-left-invariant complex structures on six-dimensional compact solvmanifolds, Differential Geom. Appl. 28 (2010), no. 2, 220-227.

[23] K. Hasegawa, Complex and Kähler structures on compact solvmanifolds, J. Symplectic Geom. 3 (2005), $749-$ 767.

[24] N.J. Hitchin, Hypersymplectic quotients, Acta Acad. Sci. Tauriensis 124 (1990), 169-180.

[25] S. Karigiannis, N.C. Leung, Hodge theory for $G_{2}$-manifolds: intermediate Jacobians and Abel-Jacobi maps, Proc. London Math. Soc. 99 (2009), 297-325.

[26] K. Kodaira, D.C. Spencer, On deformations of complex analytic structures. III. Stability theorems for complex structures, Ann. of Math. (2) 71 (1960) 43-76.

[27] K. Kodaira, Complex Manifolds and Deformations of Complex Structures, Grundlehren der Math. Wiss. 283, Springer (1986).

[28] A. Lamari, Courants kählériens et surfaces compactes, Ann. Inst. Fourier, Grenoble 49 (1999), $263-285$. 
[29] A. Latorre, L. Ugarte, Cohomological decomposition of compact complex manifolds and holomorphic deformations, Proc. Amer. Math. Soc. 145 (2017), 335-353.

[30] A. Latorre, L. Ugarte, R. Villacampa, On the Bott-Chern cohomology and balanced Hermitian nilmanifolds, Internat. J. Math. 25 (2014), no. 6, 1450057, 24 pp.

[31] A. Latorre, L. Ugarte, R. Villacampa, The ascending central series of nilpotent Lie algebras with complex structure, Trans. Amer. Math. Soc. 372 (2019), 3867-3903.

[32] T.-J. Li, W. Zhang, Comparing tamed and compatible symplectic cones and cohomological properties of almost complex manifolds, Comm. Anal. Geom. 17 (2009), 651-683.

[33] I.A. Mal'cev, A class of homogeneous spaces, Amer. Math. Soc. Transl. 39 (1951).

[34] Y. Miyaoka, Kähler metrics on elliptic surfaces, Proc. Japan Acad. 50 (1974), 533-536.

[35] I. Nakamura, Complex parallelisable manifolds and their small deformations, J. Differential Geometry 10 (1975), 85-112.

[36] K. Nomizu, On the cohomology of compact homogeneous spaces of nilpotent Lie groups, Ann. Math. 59 (1954), 531-538.

[37] G.P. Ovando, Invariant pseudo-Kähler metrics in dimension four, J. Lie Theory 16 (2006), 371-391.

[38] R.S. Palais, T.E. Stewart, Torus bundles over a torus, Proc. Amer. Math. Soc. 12 (1961), 26-29.

[39] J. Petean, Indefinite Kähler-Einstein metrics on compact complex surfaces, Commun. Math. Phys. 189 (1997), 227-235.

[40] S. Rollenske, Lie-algebra Dolbeault-cohomology and small deformations of nilmanifolds, J. Lond. Math. Soc. (2) 79 (2009), no. 2, 346-362.

[41] S.M. Salamon, Complex structures on nilpotent Lie algebras, J. Pure Appl. Algebra 157 (2001), 311-333.

[42] M. Schweitzer, Autour de la cohomologie de Bott-Chern, Prépublication de l'Institut Fourier no. 703 (2007), arXiv:0709.3528v1 [math.AG].

[43] Y.-T. Siu, Every K3 surface is Kähler, Invent. Math. 73 (1983), 139-150.

[44] J. Streets, G. Tian, A parabolic flow of pluriclosed metrics, Int. Math. Res. Not. 2010, 3101-3133.

[45] A. Teleman, The pseudo-effective cone of a non-Kählerian surface and applications, Math. Ann. 335 (2006), 965-989.

[46] W.P. Thurston, Some simple examples of symplectic manifolds, Proc. Amer. Math. Soc. 55 (1976), $467-468$.

[47] G. Tian, Smoothness of the universal deformation space of compact Calabi-Yau manifolds and its PeterssonWeil metric, Mathematical aspects of string theory (San Diego, Calif., 1986), 629-646, Adv. Ser. Math. Phys., 1, World Sci. Publishing, Singapore, 1987.

[48] A.N. Todorov, The Weil-Petersson geometry of the moduli space of $\mathrm{SU}(n=3)$ (Calabi-Yau) manifolds. I, Commun. Math. Phys. 126 (1989), 325-346.

[49] L. Ugarte, Hermitian structures on six-dimensional nilmanifolds, Transform. Groups 12 (2007), no. 1, 175-202.

[50] T. Yamada, A pseudo-Kähler structure on a nontoral compact complex parallelizable solvmanifold, Geom. Dedicata 112 (2005), 115-122.

[51] S. Yang, On blow-ups and resolutions of Hermitian-symplectic and strongly Gauduchon metrics, Arch. Math. (Basel) 104 (2015), 441-450.

(A. Latorre) Departamento de Matemática Aplicada, Universidad Politécnica de Madrid, C/ José Antonio Novais 10, 28040 Madrid, Spain

E-mail address: adela.latorre@upm.es

(L. Ugarte) Departamento de Matemáticas - I.U.M.A., Universidad de Zaragoza, Campus Plaza San Francisco, 50009 Zaragoza, Spain

E-mail address: ugarte@unizar.es 